\title{
HIGHER-ORDER MOVING MESH METHODS FOR PDE-CONSTRAINED SHAPE OPTIMIZATION*
}

\author{
ALBERTO PAGANINI ${ }^{\dagger}$, FLORIAN WECHSUNG $^{\dagger}$, AND PATRICK E. FARRELL ${ }^{\dagger}$
}

\begin{abstract}
We present a new approach to discretizing shape optimization problems that generalizes standard moving mesh methods to higher-order mesh deformations and that is naturally compatible with higher-order finite element discretizations of PDE-constraints. This shape optimization method is based on discretized deformation diffeomorphisms and allows for arbitrarily high resolution of shapes with arbitrary smoothness. Numerical experiments show that it allows the solution of PDE-constrained shape optimization problems to high accuracy.
\end{abstract}

Key words. shape optimization, PDE-constraint, finite elements, moving mesh

AMS subject classifications. 49Q10, 65N30

1. Introduction. Shape optimization problems are optimization problems where the control to be optimized is the shape of a domain. Their basic formulation generally reads

$$
\text { find } \Omega^{*} \in \underset{\Omega \in \mathcal{U}_{\mathrm{ad}}}{\operatorname{argmin}} \mathcal{J}(\Omega) \text {, }
$$

where $\mathcal{U}_{\text {ad }}$ denotes a collection of admissible shapes and $\mathcal{J}: \mathcal{U}_{\mathrm{ad}} \rightarrow \mathbb{R}$ represents a shape functional. In many applications, the shape functional depends not only on the shape of a domain $\Omega \subset \mathbb{R}^{d}$, but also on the solution $u$ of a boundary value problem (BVP) posed on $\Omega$, in which case (1) becomes

$$
\begin{gathered}
\text { find } \Omega^{*} \in \underset{\Omega \in \mathcal{U}_{\text {ad }}}{\operatorname{argmin}} \mathcal{J}\left(\Omega, u_{\Omega}\right) \quad \text { subject to } \\
u_{\Omega} \in V(\Omega), \quad a_{\Omega}\left(u_{\Omega}, v\right)=f_{\Omega}(v) \quad \text { for all } v \in W(\Omega),
\end{gathered}
$$

where (2b) represents the variational formulation of a BVP that acts as a PDEconstraint.

These problems are said to be $P D E$-constrained and are notoriously difficult to solve because the dependence of $\mathcal{J}$ on the domain is nonconvex. Additionally, the function $u_{\Omega}$ cannot be computed analytically. Even approximating it with a numerical method is challenging because the computational domain of the PDE-constraint is the unknown variable to be solved for in the shape optimization problem.

The literature abounds with numerical methods for BVPs. Here, we consider approximation by means of finite elements, which has become the most popular choice for PDE-constrained shape optimization due to its flexibility for engineering applications. Nevertheless, it is worth mentioning that alternatives based on other discretizations have also been considered $[6,9,18,43]$.

Most commonly, PDE-constrained shape optimization problems are formulated with the aim of further improving the performance of an initial design $\Omega^{0}$. The standard procedure to pursue this goal is to iteratively update some parametrization of $\Omega^{0}$ to decrease the value of $\mathcal{J}$. Obviously, the choice of this parametrization has

\footnotetext{
*Submitted to the editors DATE.

${ }^{\dagger}$ University of Oxford (paganini@maths.ac.ox.uk, wechsung@maths.ac.ox.uk, farrellp@maths.ac.ox.uk).
} 
an enormous influence on the design of the related shape optimization algorithm and on the search space $\mathcal{U}_{\text {ad }}$ itself.

In this work, we parametrize shapes by applying deformation diffeomorphisms to the initial guess $\Omega^{0}$. In this framework, solving a shape optimization problems translates into constructing an optimal diffeomorphism. To construct this diffeomorphism with numerical methods, we introduce a discretization of deformation vector fields. This approach can be interpreted as a higher-order generalization of standard moving mesh methods and can be combined with isoparametric finite elements to obtain a higher-order discretization of the PDE-constraint.

There are several advantages to using higher-degree and smoother transformations. First, higher-degree parametrization of domains allows for the consideration of more general shapes (beyond polytopes). Secondly, the efficiency of a higher-order discretization of a BVP hinges on the regularity of its solution, which depends on the regularity of the computational domain, among other factors. Finally, a smoother discretization of deformation vector fields allows the computation of more accurate Riesz representatives of shape derivatives [31,40], and thus, more accurate descent directions for shape optimization algorithms.

Our approach is generic and allows for the discretization of domain transformations based on B-splines, Lagrangian finite elements, or harmonic functions, among others. This discretization can comprise arbitrarily many basis functions and thus allow for arbitrarily high resolution of shapes with arbitrary smoothness. Moreover, because our approach decouples the discretization of the state and the control, it is straightforward to implement, and requires typically no modification of existing finite element software. This is a significant advantage for practical applications.

The rest of this article is organized as follows. In section 2, we describe how we model the search space $\mathcal{U}_{\text {ad }}$ with deformation diffeomorphisms and discuss the advantages and disadvantages of this choice. In section 3 , we give a brief introduction to shape calculus and explain how to compute steepest-descent updates for deformation diffeomorphisms using shape derivatives of shape functionals. In section 4 , we emphasize that having a PDE constraint necessitates the solution of a BVP and its adjoint at each step of the optimization, and comment on the error introduced by their finite element discretizations. In section 5, we give an introduction to isoparametric finite elements and explain why it is natural to employ this kind of discretization to approximate the state variable $u_{\Omega}$ when the domain $\Omega$ is modified by a diffeomorphism. In section 6 , we examine implementation aspects of the algorithm resulting from section 3 and section 5. In particular, we give detailed remarks for an efficient implementation of a decoupled discretization of the state and control variables. Finally, in section 7 , we perform numerical experiments. On the one hand, we consider a well-posed test case and investigate the impact of the discretization of the state and of the control variables on the performance of higher-order moving mesh methods, showing that these methods can be employed to solve PDE-constrained shape optimization problems to high accuracy. On the other hand, we consider more challenging PDE-constrained shape optimization problems and show that the proposed shape optimization method is not restricted to a specific problem.

REMARK 1. Shape optimization problems with a distinction between computational domain and control variable also exist. For instance, this is the case for PDEconstrained optimal control problems where the control is a piecewise constant coefficient in the PDE-constraint [35,39], in which case the control is the shape of the contour levels of the piecewise constant coefficient. The approach suggested in this 
work covers already this more general type of shape optimization problem. However, to simplify the discussion and reduce the amount of technicalities, we consider only problems of the form (2).

2. Parametrization of shapes via diffeomorphisms. Among the many possibilities for defining $\mathcal{U}_{\text {ad }}$, we choose to construct it by collecting all domains that can be obtained by applying (sufficiently regular) geometric transformations ${ }^{1}$ to an initial domain $\Omega^{0}$, that is,

$$
\mathcal{U}_{\text {ad }}:=\left\{T\left(\Omega^{0}\right): T \in \mathcal{T}_{\text {ad }}\right\},
$$

where $\mathcal{T}_{\text {ad }}$ is (a subgroup of) the group of $W^{1, \infty}$-diffeomorphisms. We recall that $W^{1, \infty}\left(\mathbb{R}^{d} ; \mathbb{R}^{d}\right)$ is the Sobolev space of locally integrable vector fields with essentially bounded weak derivatives. We impose this regularity requirement on $\mathcal{T}_{\text {ad }}$ to guarantee that the state constraint $(2 \mathrm{~b})$ is well-defined for every domain in $\mathcal{U}_{\text {ad }}$ (assuming that it is well-defined on the initial domain $\Omega^{0}$ ). Note that it may be necessary to strengthen the regularity requirements on $\mathcal{T}_{\text {ad }}$ if the PDE-constraint is a BVP of higher order such as, for example, the biharmonic equation [10].

While there are many alternatives (for instance level sets [2] or phase fields [22]), we prefer to describe $\mathcal{U}_{\text {ad }}$ as in (3) because it incorporates an explicit description of the boundaries of the domains contained within it. In fact, describing shapes via diffeomorphisms is a standard approach in shape optimization; cf. [15, Chap. 3]. From a theoretical point of view, it is possible to impose a metric on (3) and to investigate the existence of optimal solutions within this framework [37]. Recently, the convergence of Newton's method in this framework has also been investigated [48].

REMARK 2. The representation of domains via transformations in (3) is not unique, and it is generally possible to find two transformations $T_{1} \in \mathcal{T}_{\text {ad }}$ and $T_{2} \in \mathcal{T}_{\text {ad }}$ such that $T_{1} \neq T_{2}$ and $T_{1}\left(\Omega^{0}\right)=T_{2}\left(\Omega^{0}\right)$. For instance, this is the case if $\Omega^{0}$ is a ball and $T_{2}=T_{1} \circ T_{R}$, where $T_{R}$ is a rotation around the center of $\Omega^{0}$. To obtain a one-to-one correspondence between shapes and transformations, one can introduce equivalence classes, but this is not particularly relevant for this work.

To shorten the notation, we introduce the reduced functional

$$
j: \mathcal{U}_{\mathrm{ad}} \rightarrow \mathbb{R}, \quad \Omega \mapsto \mathcal{J}\left(\Omega, u_{\Omega}\right),
$$

which is well-defined under the following assumption on the PDE-constraint (2b).

Assumption 1. Henceforth, we assume that the BVP (2b) that acts as PDEconstraint is well-defined in the sense of Hadamard: for every $\Omega \in \mathcal{U}_{\mathrm{ad}}$ the $B V P(2 \mathrm{~b})$ has a unique solution $u_{\Omega}$ that depends continuously on the BVP data.

In section 1 , we mentioned that shape optimization problems are solved updating iteratively some parametrization of $\Omega^{0}$, that is, constructing a sequence of domains $\left\{\Omega^{(k)}\right\}_{k \in \mathbb{N}}$ so that $\left\{j\left(\Omega^{(k)}\right)\right\}_{k \in \mathbb{N}}$ decreases monotonically. For simplicity, we relax the terminology and call such a sequence minimizing, although the equality

$$
\lim _{k \rightarrow \infty} j\left(\Omega^{(k)}\right)=\inf _{\Omega \in \mathcal{U}_{\mathrm{ad}}} j(\Omega) .
$$

may not be satisfied.

When the search space $\mathcal{U}_{\text {ad }}$ is constructed as in (3), computing $\left\{\Omega^{(k)}\right\}_{k \in \mathbb{N}}$ translates into creating a sequence of diffeomorphisms $\left\{T^{(k)}\right\}_{k \in \mathbb{N}}$. Generally, the sequence $\left\{T^{(k)}\right\}_{k \in \mathbb{N}}$ is constructed according to the following procedure:

\footnotetext{
${ }^{1}$ A geometric transformation is a bijection from $\mathbb{R}^{d}$ onto itself.
} 
(a) given the current iterate $T^{(k)}$, derive a tentative iterate $\tilde{T}$,

(b) if $\tilde{T}$ satisfies certain quality criteria, set $T^{(k+1)}=\tilde{T}$ and move to the next step; otherwise compute another $\tilde{T}$.

In the next section, we discuss the computation of $\tilde{T}$ with shape derivatives. For simplicity, we first assume that the state variable $u_{\Omega}$ is known analytically and restrict our considerations to the reduced functional $j$ defined in (4). The role of the PDEconstraint and the discretization of the state variable is discussed in section 4 .

3. Iterative construction of diffeomorphisms. Shape calculus offers an elegant approach for constructing a minimizing sequence of domains $\left\{\Omega^{(k)}\right\}_{k \in \mathbb{N}}$. The key tool is the derivative of the shape functional $\mathcal{J}$ with respect to shape perturbations. To give a more precise description, let us first introduce the operator

$$
J: \mathcal{T}_{\text {ad }} \rightarrow \mathbb{R}, \quad T \mapsto j\left(T\left(\Omega^{0}\right)\right) .
$$

Since $\mathcal{T}_{\text {ad }} \subset W^{1, \infty}\left(\mathbb{R}^{d} ; \mathbb{R}^{d}\right)$, which is a Banach space with respect to the norm [19, Sect. $5.2 .2]$

$$
\|T\|_{W^{1, \infty}\left(\mathbb{R}^{d} ; \mathbb{R}^{d}\right)}:=\sum_{|\alpha| \leq 1} \operatorname{ess} \sup \left\|\mathbf{D}^{\alpha} T\right\|,
$$

we can formally define the directional derivative of $J$ at $T \in \mathcal{T}_{\text {ad }}$ in the direction $\mathcal{T} \in W^{1, \infty}\left(\mathbb{R}^{d} ; \mathbb{R}^{d}\right)$ through the limit

$$
d J(T ; \mathcal{T}):=\lim _{s \rightarrow 0^{+}} \frac{J((\mathcal{I}+s \mathcal{T}) \circ T)-J(T)}{s}=\lim _{s \rightarrow 0^{+}} \frac{J(T+s \mathcal{T} \circ T)-J(T)}{s} .
$$

REMARK 3. Note that $\mathcal{I}+s \mathcal{T}$ is a $W^{1, \infty}$-diffeomorphism for sufficiently small $s$ [1, Lemma 6.13].

A shape functional $\mathcal{J}$ is said to be shape differentiable (in $T\left(\Omega^{0}\right)$ ) if the corresponding functional (6) is Fréchet differentiable (in $T$ ), that is, if (8) defines a linear continuous operator on $W^{1, \infty}\left(\mathbb{R}^{d} ; \mathbb{R}^{d}\right)$ such that

$$
|J(T+s \mathcal{T} \circ T)-J(T)-d J(T ; s \mathcal{T})|=o(s) \quad \text { for all } \mathcal{T} \in W^{1, \infty}\left(\mathbb{R}^{d} ; \mathbb{R}^{d}\right) .
$$

Remark 4. Generally, Assumption 1 is not sufficient to guarantee that $\mathcal{J}$ is shape differentiable. In particular, it is necessary to ensure that the solution operator $\Omega \mapsto u_{\Omega}$ is continuously differentiable; cf. [29, Sect. 1.6].

The Fréchet derivative $d J$ can be used to construct a sequence of diffeomorphisms $\left\{T^{(k)}\right\}_{k \in \mathbb{N}}$ to solve (2) in a steepest descent fashion. More specifically, the entries of this sequence take the form

$$
T^{(0)}(\mathbf{x})=\mathbf{x} \quad \text { and } \quad T^{(k+1)}(\mathbf{x})=\left(\mathcal{I}+\mathrm{d} T^{(k)}\right) \circ\left(T^{(k)}(\mathbf{x})\right),
$$

where the update $\mathrm{d} T^{(k)}: \mathbb{R}^{d} \rightarrow \mathbb{R}^{d}$ is computed with the help of $d J$. For instance, we could define [29, Page 103]

$$
d T^{(k)} \in \underset{\substack{\mathcal{T} \in W^{1, \infty}\left(\mathbb{R}^{d} ; \mathbb{R}^{d}\right),\|\mathcal{T}\|_{W^{1, \infty}}=1}}{\operatorname{argmin}} d J\left(T^{(k)} ; \mathcal{T}\right) .
$$

Unfortunately, such a descent direction may not exist without making further assumptions on $d J$ because $W^{1, \infty}\left(\mathbb{R}^{d} ; \mathbb{R}^{d}\right)$ is not reflexive; cf. [33]. However, in [35] it has 
been shown that in many instances (and under suitable assumptions), the operator $d J$ takes the form

$$
d J\left(T^{(k)} ; \mathcal{T}\right)=\int_{T^{(k)}\left(\Omega_{0}\right)} \sum_{i, j=1}^{d} s_{1}^{i, j} \mathbf{D} \mathcal{T}^{i, j}+\sum_{\ell=1}^{d} s_{0}^{\ell} \mathcal{T}^{\ell} \mathrm{d} \boldsymbol{x},
$$

where $s_{1}^{i, j}, i, j=1, \ldots, d$ and $s_{0}^{\ell}, \ell=1, \ldots, d$ are (instance dependent) $L^{1}\left(\mathbb{R}^{d}\right)$ functions. The following proposition ${ }^{2}$ states that, in this case, (11) can be used to define steepest descent directions.

Proposition 3.1. Let $d J$ be as in (12). Then, there exists a descent direction $d T^{(k)}$ as defined in (11)

Proof. First of all, we recall that $L^{\infty}(D)$ is isometrically isomorphic to the dual $X^{*}$ of $X=L^{1}(D)$ (for any open domain $D \subset \mathbb{R}^{m}$ in any fixed dimension $m$ ). We denote by $\phi_{D}: L^{\infty}(D) \rightarrow X^{*}$ this isomorphism. The duality pairing $\langle\cdot, \cdot\rangle_{X^{*} \times X}$ can be characterized by

$$
\langle f, g\rangle_{X^{*} \times X}=\int_{D} \phi_{D}^{-1}(f) g \mathrm{~d} \boldsymbol{x} .
$$

Clearly, similar pairings exist for Cartesian products of $L^{\infty}(D)$. Finally, note that $L^{1}(D)$ is separable. Therefore, by the Banach-Alaoglu theorem, any bounded sequence in $L^{\infty}(D)$ has a subsequence $\left\{\mathbf{x}_{n}\right\}$ that converges weakly-* to an $\mathbf{x} \in L^{\infty}(D)$, that is,

$$
\lim _{n \rightarrow \infty} \int_{D} \mathbf{x}_{n} g \mathrm{~d} \boldsymbol{x}=\int_{D} \mathbf{x} g \mathrm{~d} \boldsymbol{x} \quad \text { for every } g \in L^{1}(D) .
$$

Using these results, we show that a steepest descent direction exists.

Let $\mathcal{T}_{n}$ be a minimizing sequence of (11). By definition, $\mathcal{T}_{n}$ is bounded in $W^{1, \infty}\left(\mathbb{R}^{d} ; \mathbb{R}^{d}\right)$, and hence in $L^{\infty}\left(\mathbb{R}^{d}, \mathbb{R}^{d}\right)$, too. Therefore, there exists a subsequence $\mathcal{T}_{n_{k}}$ that converges weakly-* to a $T \in L^{\infty}\left(\mathbb{R}^{d}, \mathbb{R}^{d}\right)$. Since $\mathcal{T}_{n_{k}}$ is bounded in $W^{1, \infty}\left(\mathbb{R}^{d} ; \mathbb{R}^{d}\right)$, there is a subsequence $\mathcal{T}_{n_{k_{\ell}}}$ such that $\mathbf{D} \mathcal{T}_{n_{k_{\ell}}}$ converges weakly-* in $L^{\infty}\left(\mathbb{R}^{d}, \mathbb{R}^{d, d}\right)$. By the definition of weak derivative, it is easy to see that the weakly-* limit of $\mathbf{D} \mathcal{T}_{n_{k_{\ell}}}$ is DT. This shows that $T \in W^{1, \infty}\left(\mathbb{R}^{d}, \mathbb{R}^{d}\right)$.

Since (12) is a sum of duality pairings as in (13) (with $D=\mathbb{R}^{d}$ and $g=$ $\chi_{T^{(k)}\left(\Omega_{0}\right)} s 1_{1}^{i, j}$ or $g=\chi_{T^{(k)}\left(\Omega_{0}\right)} s_{0}^{\ell}$, where $\chi_{T^{(k)}\left(\Omega_{0}\right)}$ is the characteristic function associated to $\left.T^{(k)}\left(\Omega_{0}\right)\right), d J$ is weakly-* continuous. Therefore, $T$ is a minimizer, because it is the weak-* limit of $\mathcal{T}_{n_{k_{\ell}}}$, which is a subsequence of a minimizing sequence.

Finally, to show that $\|T\|_{W^{1, \infty}\left(\mathbb{R}^{d} ; \mathbb{R}^{d}\right)}=1$, we recall that the norm of a Banach space is weak-* lower semi-continuous. Therefore,

$$
\|T\|_{W^{1, \infty}\left(\mathbb{R}^{d} ; \mathbb{R}^{d}\right)} \leq \liminf _{k \rightarrow \infty}\left\|\mathcal{T}_{n_{k}}\right\|_{W^{1, \infty}\left(\mathbb{R}^{d} ; \mathbb{R}^{d}\right)} \leq 1
$$

and since $d J\left(T^{(k)} ; \cdot\right)$ is linear, $\|T\|_{W^{1, \infty}\left(\mathbb{R}^{d} ; \mathbb{R}^{d}\right)}=1$.

To conclude, note that $d J\left(T^{(k)} ; T\right)>-\infty$ because $d J\left(T^{(k)} ; \cdot\right)$ is continuous.

Although possibly well-defined, it is challenging to compute such a descent direction $d T^{(k)}$ (because $W^{1, \infty}\left(\mathbb{R}^{d} ; \mathbb{R}^{d}\right)$ is infinite dimensional and neither reflexive

\footnotetext{
new.

${ }^{2}$ We provide a full proof of this proposition because, to the best of our knowledge, this result is
} 
nor separable). One possible remedy is to introduce a Hilbert subspace $(\mathcal{X},(\cdot, \cdot) \mathcal{X})$, $\mathcal{X} \subset W^{1, \infty}\left(\mathbb{R}^{d} ; \mathbb{R}^{d}\right)$, and to compute

$$
d T_{\mathcal{X}}^{(k)}:=\underset{\substack{\mathcal{T} \in \mathcal{X},\|\mathcal{T}\|_{\mathcal{X}}=1}}{\operatorname{argmin}} d J\left(T^{(k)} ; \mathcal{T}\right),
$$

that is, the gradient of $d J$ with respect to $(\cdot, \cdot)_{\mathcal{X}}$. Up to a scaling factor, the solution of (16) can be computed by solving the variational problem: find $d T^{(k)}$ such that

$$
\left(d T^{(k)}, \mathcal{T}\right)_{\mathcal{X}}=-d J\left(T^{(k)} ; \mathcal{T}\right) \text { for all } \mathcal{T} \in \mathcal{X},
$$

which is well-posed by the Riesz representation theorem. However, the condition $\mathcal{X} \subset W^{1, \infty}\left(\mathbb{R}^{d} ; \mathbb{R}^{d}\right)$ is restrictive (for a Hilbert space). For instance, the general Sobolev inequalities guarantee that the Sobolev space $H^{k}\left(\mathbb{R}^{d} ; \mathbb{R}^{d}\right)$ is contained in $W^{1, \infty}\left(\mathbb{R}^{d} ; \mathbb{R}^{d}\right)$ only for $k \geq d / 2+1$ [19, Sect. 5.6.3].

A more popular approach is to introduce a finite dimensional subspace $Q_{N} \subset$ $\mathcal{X} \cap W^{1, \infty}\left(\mathbb{R}^{d} ; \mathbb{R}^{d}\right)$ and to compute the solution of

$$
\left(d T_{N}^{(k)}, \mathcal{T}_{N}\right)_{\mathcal{X}}=-d J\left(T^{(k)} ; \mathcal{T}_{N}\right) \quad \text { for all } \mathcal{T}_{N} \in Q_{N}
$$

In this case, the requirement $\mathcal{X} \subset W^{1, \infty}\left(\mathbb{R}^{d} ; \mathbb{R}^{d}\right)$ can be dropped as long as the dimension $N:=\operatorname{dim}\left(Q_{N}\right)$ of $Q_{N}$ is finite. However, note that if $\left\{Q_{N}\right\}_{N \in \mathbb{N}}$ is a family of nested finite dimensional spaces such that $\overline{\cup_{N \in \mathbb{N}} Q_{N}} \mathcal{X}=\mathcal{X}$, the sequence $d T_{N}^{(k)}$ can be interpreted as the Ritz-Galerkin approximation of $d T^{(k)}$. Therefore, as $N \rightarrow \infty$, the sequence $d T_{N}^{(k)}$ may converge to an element of $\mathcal{X} \backslash W^{1, \infty}\left(\mathbb{R}^{d} ; \mathbb{R}^{d}\right)$, which does not qualify as an admissible update.

The trial space $Q_{N}$ can be constructed with linear Lagrangian finite elements defined on (a mesh of) a hold-all domain $D \supset \Omega^{0}$. The resulting algorithm is equivalent to standard moving mesh methods [40]. Alternatively, one can employ tensorized B-splines [30]. Lagrangian finite elements have the advantage of inclusion in standard finite element software, whereas B-splines offer higher regularity, which is often desirable (as we will argue in section 6). For instance, univariate B-splines of degree $d$ are in $W^{d, \infty}(\mathbb{R})[32]$, whereas Lagrangian finite elements are not even $C^{1}$. As for the Hilbert space $\mathcal{X}$, one usually opts for $\mathcal{X}=H^{1}(D)$ or, equivalently, for $H^{1 / 2}\left(\partial \Omega^{(k)}\right)$ combined with an elliptic extension operator onto $D$ [45]. This choice can be motivated by considerations of the shape Hessian [18,45].

To the best of our knowledge, it has not been settled yet which definition of steepest direction among (11), (16), and (18) is best suited to formulate a numerical shape optimization algorithm. Since the focus of this work is more on the discretization of shape optimization problems than on actual optimization algorithms, we postpone investigations of this topic to future research. In our numerical experiments in section 7, we will employ (18), which is the computationally most tractable definition. However, note that computing steepest directions according to (11) or (16) would also inevitably involve some discretization, because $W^{1, \infty}\left(\mathbb{R}^{d} ; \mathbb{R}^{d}\right.$ ) (and generally $\mathcal{X}$ ) are infinite dimensional.

We conclude this section with the Hadamard homeomorphism theorem [34, Thm 1.2], which gives explicit criteria to verify that the entries of the sequence $\left\{T^{(k)}\right\}_{k \in \mathbb{N}}$ defined in (10) are admissible transformations.

Theorem 3.2. Let $X$ and $Y$ be finite dimensional Euclidean spaces, and let $T$ : $X \rightarrow Y$ be a $C^{1}$-mapping that satisfies the following conditions: 
1. $\operatorname{det}(\mathbf{D} T)(x) \neq 0$ for all $x \in X$.

2. $\|T(x)\| \rightarrow \infty$ as $\|x\| \rightarrow \infty$.

Then $T$ is a $C^{1}$-diffeomorphism from $X$ to $Y$.

A counterpart of Theorem 3.2 for $W^{1, \infty}$-transformations can be found in [21].

For the sequence (10), note that the second hypothesis of Theorem 3.2 is automatically satisfied if the hold-all domain $D$ is bounded, because the update $\mathrm{d} T^{(k)}$ has compact support, and $T^{(k+1)}(\mathbf{x})=\mathbf{x}$ for every $\mathbf{x} \in \mathbb{R}^{d} \backslash \bar{D}$.

4. Shape derivatives of PDE-constrained functionals. To simplify the exposition, in the previous section we treated the dependence of $J$ on $u$ implicitly; this dependence was hidden in the reduced functional $j$. We now examine the consequences of this dependence, as it introduces additional difficulties. Indeed, it is generally the case that to evaluate the Fréchet derivative $d J$ it is necessary to solve (at least) one BVP. To illustrate this fact, we consider the following example:

$$
\begin{gathered}
\mathcal{J}\left(\Omega, u_{\Omega}\right)=\frac{1}{2} \int_{\Omega} u_{\Omega}^{2} \mathrm{~d} \boldsymbol{x}, \quad \text { subject to } \\
u_{\Omega} \in H^{1}(\Omega), \quad \int_{\Omega} \nabla u_{\Omega} \cdot \nabla v+u_{\Omega} v \mathrm{~d} \boldsymbol{x}=\int_{\Omega} v \mathrm{~d} \boldsymbol{x} \quad \text { for all } v \in H^{1}(\Omega) .
\end{gathered}
$$

Its shape derivative reads [39, Eq. 2.12]

$$
\begin{aligned}
d J(T ; \mathcal{T})=\int_{T\left(\Omega^{0}\right)}( & \nabla u_{T\left(\Omega^{0}\right)} \cdot\left(\mathbf{D} \mathcal{T}+\mathbf{D} \mathcal{T}^{\top}\right) \nabla p \\
& \left.+\left(p+u_{T\left(\Omega^{0}\right)}^{2}-\nabla u_{T\left(\Omega^{0}\right)} \cdot \nabla p-u_{T\left(\Omega^{0}\right)} p\right) \operatorname{div} \mathcal{T}\right) \mathrm{d} \boldsymbol{x}
\end{aligned}
$$

where $p \in H^{1}\left(T\left(\Omega^{0}\right)\right)$ is the solution of the adjoint BVP

$$
\int_{T\left(\Omega^{0}\right)} \nabla p \cdot \nabla v+p v \mathrm{~d} \boldsymbol{x}=\int_{T\left(\Omega^{0}\right)} u_{T\left(\Omega^{0}\right)} v \mathrm{~d} \boldsymbol{x} \quad \text { for all } v \in H^{1}\left(T\left(\Omega^{0}\right)\right) .
$$

Formula (20) clearly shows that it is necessary to compute the functions $u_{T\left(\Omega^{0}\right)}$ and $p$ to evaluate $d J$. The adjoint BVP (21) is introduced to derive a formula of $d J$ that does not contain the shape derivative of $u_{\Omega}$. This is a well-known strategy in PDE-constrained optimization [29, Sect. 1.6].

In general, deriving explicit formulae for Fréchet derivatives of PDE-constrained functionals is a delicate and error prone task. However, in many instances one can introduce a Lagrangian functional that allows the automation of the differentiation process and gives the correct adjoint equations [29, Sect. 1.6.4]. The level of automation is such that numerical software is capable of differentiating several PDEconstrained functionals [20]. Clearly, Lagrangians are useful also for the special case of PDE-constrained shape functionals [15, Chap. 10], and dedicated numerical software for shape differentiation has recently become available [42].

Remark 5. The Hadamard-Zolésio structure theorem [15, Chap. 9, Thm 3.6] states that, under certain regularity assumptions on $\Omega$, the Fréchet derivative $d J(\Omega ; \mathcal{T})$ depends only on perturbations $\mathcal{T}(\partial \Omega)$ of the domain boundary. As a consequence, the derivative of most shape functionals can be formulated as an integral both in the volume $\Omega$ and on the boundary $\partial \Omega$, and these formulations are equivalent. For instance, the boundary formulation that corresponds to (20) reads [39, Eq. 2.13]

$$
\int_{T\left(\partial \Omega^{0}\right)} \mathcal{T} \cdot \mathbf{n}\left(u_{T\left(\Omega^{0}\right)}^{2}-\nabla u_{T\left(\Omega^{0}\right)} \cdot \nabla p-u_{T\left(\Omega^{0}\right)} p+p\right) \mathrm{d} S .
$$


When the state and the adjoint variables are replaced by numerical approximations, these two formulae define two different approximations of $d J$. In the framework of finite elements, it has been shown that volume based formulations usually offer higher accuracy compared to their boundary based counterparts [31,38]. Additionally, the combination of volume based formulae with piecewise linear finite element discretization of the control variable results in shape optimization algorithms for which the paradigms optimize-then-discretize and discretize-then-optimize commute [11,30]. This does not hold in general for boundary based formulae because piecewise linear finite elements do not fulfill the necessary regularity requirements, and the equivalence of boundary and volume based formulae is not guaranteed [11].

5. Isoparametric Lagrangian finite elements. To evaluate the shape functional $\mathcal{J}\left(\Omega, u_{\Omega}\right)$, it is necessary to approximate the function $u_{\Omega}$, which is the solution of the PDE-constraint $(2 \mathrm{~b})$. For the Fréchet derivative $d J$, it may be necessary to also approximate the solution $p$ of an adjoint BVP. In this work, we consider the discretization of $(2 \mathrm{~b})$ and the adjoint BVP by means of finite elements. Finite element spaces are defined on meshes of the computational domain. As shape optimization algorithms modify the computational domain, a new mesh is required at each iteration. This new mesh can either be constructed de novo or by modifying a previously existing mesh. On the one hand, remeshing should be avoided because it is computationally expensive and may introduce undesirable noise in the optimization algorithm. On the other hand, updating the mesh is a delicate process and may return a mesh with poor quality (which in turn introduces noise in the optimization as well).

Isoparametric finite elements offer an interesting perspective on the process of mesh updating that fits well with our encoding of changes in the domain via geometric transformations. In particular, with isoparametric finite elements it is possible to mimic the modification of the computational domain without tampering directly with the finite element mesh. Additionally, isoparametric finite element theory provides insight into the extent to which remeshing can be avoided. Next, we provide a concise recapitulation of isoparametric finite element theory. For simplicity, we assume that the PDE-constraint is a linear $V$-elliptic second-order BVP. However, we believe that most of the considerations readily cover more general BVPs. For a more thorough introduction to isoparametric finite elements, we refer to [13, Sect. 4.3].

The Ritz-Galerkin discretization of $(2 \mathrm{~b})$ reads

$$
\text { find } \quad u_{h} \in V_{h}(\Omega), \quad a_{\Omega}\left(u_{h}, v_{h}\right)=f_{\Omega}\left(v_{h}\right) \quad \text { for all } v_{h} \in V_{h}(\Omega),
$$

where $V_{h}(\Omega)$ is a finite dimensional subspace of $V(\Omega)=W(\Omega)$. Henceforth, we restrict ourselves to Lagrangian finite element approximations on simplicial meshes.

Let us assume for the moment that $\Omega$ is a polytope. The most common construction of finite element spaces begins with a triangulation $\Delta_{h}(\Omega)$ of $\Omega$. This triangulation is used to introduce global basis functions that span the finite element space. The finite element space is called Lagrangian if the degrees of freedom of its global basis functions are point evaluations [13, Page 36], and it is called of degree $p$ if the local basis functions, that is, the restriction of global basis functions to elements $K$ of the triangulation, are polynomials of degree $p$.

It is well known that Lagrangian finite elements on simplicial meshes are affine equivalent. Affine equivalence means that we can define a reference element $\hat{K}$ and a set of reference local basis functions $\left\{\hat{b}_{i}\right\}_{i \leq M}$ on $\hat{K}$, and construct a family of affine diffeomorphisms $\left\{G_{K}: \hat{K} \rightarrow K\right\}_{K \in \Delta_{h}(\Omega)}$ such that the local basis functions $\left\{b_{i}^{K}\right\}_{i \leq M}$ on $K$ satisfy $b_{i}^{K}(\mathbf{x})=\hat{b}_{i}\left(G_{K}^{-1}(\mathbf{x})\right)$. Note that both $\left\{b_{i}^{K}\right\}_{i \leq M}$ and $\left\{\hat{b}_{i}\right\}_{i \leq M}$ 
are polynomials, because the pullback induced by a bijective affine transformation is an automorphism.

Issues with this construction arise if $\Omega$ has curved boundaries. In this case, we introduce first an affine equivalent finite element space $V_{h}(\Omega)$ built on the triangulation $\Delta_{h}(\Omega)$ of a polytope $\Omega$ that approximates $\Omega$. Then, we construct a vector field $F \in\left(V_{h}(\Omega)\right)^{d}$ such that $F(\partial \Omega) \approx \partial \Omega$ and generate a (curved) triangulation $\Delta_{h}(\Omega)$ by deforming the elements of $\Delta_{h}(\Omega)$ according to $F$. Finally, we define the finite element space $V_{h}(\Omega)$ on $\Delta_{h}(\Omega)$ by choosing $b_{i}^{K}(\mathbf{x})=\hat{b}_{i}\left(G_{K}^{-1}\left(F^{-1}(\mathbf{x})\right)\right)$ as local basis functions. This construction leads to so-called isoparametric finite elements. Again, this space is called Lagrangian if the reference local basis functions $\left\{\hat{b}_{i}\right\}_{i \leq M}$ are polynomials. However, note that the local basis functions $\left\{b_{i}\right\}_{i \leq M}$ of isoparametric Lagrangian finite elements may not be polynomials.

Isoparametric Lagrangian finite elements on curved domains are proved to retain the approximation properties of Lagrangian finite elements on polytopes under the following additional assumptions [13, Thm 4.3.4]:

1. the triangulation $\Delta_{h}(\Omega)$ is regular [13, Page 124],

2 . the mesh width $h$ is sufficiently small,

3. for every quadrature point $\mathbf{x}_{q} \in \hat{K}$, and for every element $K \in \Delta_{h}(\Omega)$

$$
\left\|F\left(G_{K}\left(\mathbf{x}_{q}\right)\right)-G_{K}\left(\mathbf{x}_{q}\right)\right\|=\mathcal{O}\left(h^{p}\right),
$$

and $F\left(G_{K}\left(\mathbf{x}_{q}\right)\right) \in \partial \Omega$ whenever $G_{K}\left(\mathbf{x}_{q}\right) \in \partial \Omega$.

Equation (24) is sufficient to guarantee that the map $F \circ G_{K}$ is a diffeomorphism, and to provide algebraic estimates of the form

$$
\left\|\mathbf{D}^{\alpha}\left(F \circ G_{K}\right)\right\|=\mathcal{O}\left(h^{\alpha}\right),
$$

which are necessary to derive the desired approximation estimates.

This knowledge of isoparametric finite elements is sufficient to tackle our initial problem: solve (23) on $\Omega^{(k)}$ (where $\Omega^{(k)}:=T^{(k)}\left(\Omega^{0}\right)$ ).

In the first iteration, we construct $V_{h}\left(\Omega^{0}\right)$ in the isoparametric fashion described above. First, we generate a triangulation of a suitable polytope $\Omega^{0}$ that approximates $\Omega^{0}$. Then, we define the finite element space $V_{h}\left(\Omega^{0}\right)$ and generate a transformation $F^{(0)} \in\left(V_{h}\left(\Omega^{0}\right)\right)^{d}$ that maps $\Omega^{0}$ onto $\Omega^{0}$. Finally, we construct $V_{h}\left(\Omega^{0}\right)$ by combining reference local basis functions with the diffeomorphism $F^{(0)}$.

In the next iteration, we construct $V_{h}\left(\Omega^{(1)}\right)$ in the same way, but replacing the diffeomorphism $F^{(0)}$ with the interpolant

$$
\left(V_{h}\left(\Omega^{0}\right)\right)^{d} \ni F^{(1)}:=\mathcal{I}_{h}\left(T^{(1)} \circ F^{(0)}\right),
$$

where $\mathcal{I}_{h}$ denotes the interpolation operator onto $\left(V_{h}\left(\Omega^{0}\right)\right)^{d}$. Since

$$
T^{(1)}(\mathbf{x})=\mathbf{x}+\mathrm{d} T^{(0)}(\mathbf{x})
$$

the map $F^{(1)}$ can be written as

$$
F^{(1)}=F^{(0)}+\mathcal{I}_{h}\left(\mathrm{~d} T^{(0)} \circ F^{(0)}\right) .
$$

Repeating this procedure at every iteration results in the isoparametric space $V_{h}\left(\Omega^{(k)}\right)$ being constructed with the map

$$
\begin{aligned}
F^{(k)} & =\mathcal{I}_{h}\left(T^{(k)} \circ F^{(0)}\right), \\
& =\mathcal{I}_{h}\left(T^{(k-1)} \circ F^{(0)}\right)+\mathcal{I}_{h}\left(\mathrm{~d} T^{(k-1)} \circ T^{(k-1)} \circ F^{(0)}\right), \\
& =F^{(k-1)}+\mathcal{I}_{h}\left(\mathrm{~d} T^{(k-1)} \circ F^{(k-1)}\right),
\end{aligned}
$$


where the second equality follows from $F^{(k-1)}=\mathcal{I}_{h}\left(T^{(k-1)} \circ F^{(0)}\right)$.

In general, the map $F^{(k)}$ may not fulfill the condition (24). However, by $W^{1, \infty}$ error estimates of $\mathcal{I}_{h}$ [13, Thm 4.3.4], it holds that

$$
\operatorname{det}\left(\mathbf{D} F^{(k)}\right)(x) \rightarrow \operatorname{det}\left(\mathbf{D}\left(T^{(k)} \circ F^{(0)}\right)\right)(x) \text { as } h \rightarrow 0 .
$$

This, in light of Theorem 3.2, guarantees that $F^{(k)}$ is indeed a diffeomorphism if $h$ is small enough (because $T^{(k)} \circ F^{(0)}$ is a diffeomorphism as well, and therefore $\left.\operatorname{det}\left(\mathbf{D}\left(T^{(k)} \circ F^{(0)}\right)\right)(x) \neq 0\right)$. Additionally, note that the element transformation $G_{K}: \hat{K} \rightarrow K$ is affine, and thus,

$$
\mathbf{D}^{\alpha}\left(F^{(k)} \circ G_{K}\right)=\left(\mathbf{D}^{\alpha}\left(F^{(k)}\right) \circ G_{K}\right)\left(\mathbf{D} G_{K}\right)^{\alpha} .
$$

Therefore,

$$
\left\|\mathbf{D}^{\alpha}\left(F^{(k)} \circ G_{K}\right)\right\| \leq\left\|\left(\mathbf{D}^{\alpha}\left(F^{(k)}\right) \circ G_{K}\right)\right\|\left\|\left(\mathbf{D} G_{K}\right)^{\alpha}\right\| \leq\left\|\mathbf{D}^{\alpha}\left(F^{(k)}\right)\right\| h^{\alpha} .
$$

The estimate (27) is asymptotically equivalent to (25). This implies that modifying the transformation used to generated the isoparametric finite element space does not affect its approximation properties as long as $\left\|\mathbf{D}^{\alpha}\left(F^{(k)}\right)\right\|$ is moderate.

REMARK 6. It is not strictly necessary to replace the transformation $T^{(k)} \circ F^{(0)}$ with its interpolant $F^{(k)}$. However, evaluating $T^{(k)}$ can be computationally expensive and may not be supported natively in finite element software (in particular, if evaluating $T^{(k)}$ involves a complicated formula). On the other hand, as explained in the section $3, T^{(k)}$ lies in practice in a finite dimensional space. Therefore, the interpolation operator $\mathcal{I}_{h}$ can be represented as a matrix, which can be used to significantly speed up the computation of the update $d T^{(k)}$, as explained in section 6 .

REMARK 7. Usually, the isoparametric transformation $F$ is chosen to be the identity on elements of $\Delta_{h}(\Omega)$ that do not share edges/faces with $\partial \Omega$. This particular choice of $F$ is made to decrease the computational cost of matrix/vector assembly, and is not dictated by error analysis. In our approach, the function $F$ will generally differ from the identity even in the interior of the domain.

REMARK 8. In [30], the authors suggest to purse shape optimization in Lagrangian coordinates by reformulating shape optimization problems as an optimal control problem on the initial domain. The resulting method is formally equivalent to the one presented in this work, but implies hard-coding of geometric transformation into shape functionals and PDE-constraints (which is problem dependent), and requires the derivation of Fréchet derivatives in Lagrangian coordinates (which are usually not considered in the shape optimization literature). In contrast, the approach presented in this work exploits the fact that these geometric transformations are included in standard finite element software, and allows the use of formulae for Fréchet derivatives that are already available in the literature.

6. Implementation aspects. The previous sections consider different discretization aspects of the shape optimization problem (2). Section 3 introduces the finite dimensional space $Q_{N}$ to construct the sequence of diffeomorphisms (10). Section 5, on the other hand, introduces the finite dimensional space $\left(V\left(\Omega^{0}\right)\right)^{d}$ to approximate the solution of $(2 \mathrm{~b})$ by means of isoparametric finite elements.

There are conflicting demands on the choice of these two finite dimensional spaces. On the one hand, employing the same discretization based on piecewise linear Lagrangian finite elements greatly simplifies the implementation in existing finite element libraries and may reduce the execution time. On the other hand, a decoupled 
discretization facilitates enforcing stability in the optimization process. For instance, the authors of [3,24] suggest the use of linear Lagrangian finite elements built on two nested meshes: a coarser one to discretize the geometry and a finer one to solve the state equation. They report that this reduces the presence of spurious oscillations in the optimized shape.

A decoupled discretization may also be required if one aims at using higher-order approximations of the state variable $u$. To elucidate this, recall that the use of higherorder finite elements is motivated only if the exact solution is sufficiently regular. More specifically, isoparametric finite element solutions of degree $p$ converge as $\mathcal{O}\left(h^{p}\right)$ in the energy norm provided that the exact solution satisfies $u \in H^{p+1}(\Omega)$, whereas the convergence rate deteriorates if $u$ is less regular [13, Thm 3.2.1] or if isoparametric finite elements are replaced by standard affine-parametric finite elements $[13, \mathrm{Rmk}$ 4.4.4 (ii)].

It is virtually impossible to prescribe universal and sharp rules that ensure that the solution of the state constraint remains sufficiently regular during the optimization process, but elliptic regularity theory can provide some guidelines. Assuming the problem data is sufficiently smooth, the solution of a linear elliptic Dirichlet BVP is $H^{s}$-regular [12, Def. 7.1] when $\Omega$ has a $C^{s}$-boundary [25, Thm 8.13] (see also [27] for an extensive treatment of elliptic regularity theory). Therefore, it might be desirable to employ sufficiently regular transformations, so that the regularity of the domain is preserved during the optimization process. In this case, typical isoparametric Lagrangian finite elements are not a good choice for $Q_{N}$ because they only allow $W^{1, \infty}$ piecewise polynomial representations of the domain transformations. The natural alternative is to employ multivariate B-splines of degree $\tilde{p}$ [32, Def. 4.1], which are piecewise polynomials with compact support and are both $W^{\tilde{p}, \infty}$ and $C^{\tilde{p}-1}$ regular.

For these reasons, we focus on the more general case of a decoupled discretization of $\left(V\left(\Omega^{0}\right)\right)^{d}$ and $Q_{N}$ and discuss the implementation details for the following simple optimization algorithm, which covers all fundamental aspects of shape optimization.

\section{Minimal shape optimization pseudo-code.}

1. initialize, then, for $k \geq 0$ :

2. compute the state $u$ and evaluate $J$; stop if converged, otherwise

3. compute the update $d T$ solving (18)

4. choose $s$ such that $T+s d T \circ T$ is feasible and $J$ is minimal

5. update $T$ and go back to step 2 .

Step 1. First, we construct the finite element space $\left(V_{h}\left(\Omega^{0}\right)\right)^{d}:=\operatorname{span}\left\{\mathbf{v}_{i}\right\}_{i=1}^{M}$ and store the coefficient vector $\mathbf{f}^{(0)} \in \mathbb{R}^{M}$ of the transformation $F^{(0)} \in\left(V_{h}\left(\Omega^{0}\right)\right)^{d}$, which maps $\Omega^{0}$ onto (an approximation of) $\Omega^{0}$. Then, we construct the space $Q_{N}:=$ $\operatorname{span}\left\{\mathbf{q}_{i}\right\}_{i=1}^{N}$, initialize the vector field $T^{(0)} \in Q_{N}$ to the identity, and store its coefficients in the vector $\mathbf{t}^{(0)} \in \mathbb{R}^{N}$. Finally, we store the matrix representation $\mathbf{I}_{h}$ of the interpolation operator

$$
\mathcal{I}_{h}: Q_{N} \rightarrow\left(V_{h}\left(\Omega^{0}\right)\right)^{d} .
$$

The matrix $\mathbf{I}_{h}$ is sparse if the basis functions $\left\{\mathbf{v}_{i}\right\}_{i=1}^{M}$ and $\left\{\mathbf{q}_{i}\right\}_{i=1}^{N}$ have (small) compact support.

Step 2. First, we compute the coefficients of $F^{(k)}:=\mathcal{I}_{h}\left(T^{(k)} \circ F^{(0)}\right)$. This is done by computing

$$
\mathbf{f}^{(k)}=\mathbf{f}^{(k-1)}+\mathbf{I}_{h} \mathbf{d} \mathbf{t}^{(k-1)},
$$


as will be justified in the next step. Then, we approximate $u_{\Omega^{(k)}}$ by means of isoparametric finite elements and evaluate $\mathcal{J}$ on the domain $\Omega^{(k)}$. If the convergence criteria are not satisfied, we proceed further and compute an update of $T^{(k)}$.

Step 3. First, we have to assemble the load vector $\mathbf{d} \tilde{\mathbf{J}}_{\mathbf{q}}^{(k)}:=\left\{d J\left(T^{(k)} ; \mathbf{q}_{i}\right)\right\}_{i=1}^{N}$. This can be computationally expensive because $d J$ depends on $u$, which is approximated with a finite element function and lives on a finite element mesh. Therefore, to evaluate $d J$ it is necessary to loop through the finite element mesh. Although one loop is sufficient if one evaluates the contribution of each cell for all basis functions in $Q_{N}$, evaluating these functions can be computationally expensive and may require extensive modifications of finite element software. Therefore, it may be desirable to employ a different strategy, which we detail below.

Let $\left\{\mathbf{v}_{i}^{(k)}\right\}_{i=1}^{M}$ denote the isoparametric basis of $\left(V_{h}\left(\Omega^{(k)}\right)\right)^{d}$. The vector

$$
\mathbf{d} \tilde{\mathbf{J}}_{\mathbf{v}^{(k)}}^{(k)}:=\left\{d J\left(T^{(k)} ; \mathbf{v}_{i}^{(k)}\right)\right\}_{i=1}^{M}
$$

can be assembled efficiently with existing software, because the basis functions $\left\{\mathbf{v}_{i}^{(k)}\right\}$ are generally included in finite element software (whilst $\left\{\mathbf{q}_{i}\right\}$ may not be). Interestingly, the product of the transpose of the interpolation matrix $\mathbf{I}_{h}^{\top}$ with (29) can be interpreted as the approximation

$$
\mathbf{I}_{h}^{\top} \mathbf{d} \tilde{\mathbf{J}}_{\mathbf{v}^{(k)}}^{(k)} \approx\left\{d J\left(T^{(k)} ; \mathbf{q}_{i} \circ\left(F^{(0)}\right)^{-1} \circ\left(T^{(k)}\right)^{-1}\right)\right\}_{i=1}^{N},
$$

where the right-hand side corresponds to the evaluation of $d J\left(T^{(k)} ; \cdot\right)$ on functions that move along with the domain transformation (see Figure 1). To explain the nature of the approximation in (30), we introduce a new finite dimensional space

$$
Q_{N}^{(k)}:=\operatorname{span}\left\{\mathbf{q}_{i}^{(k)}:=\mathbf{q}_{i} \circ\left(F^{(0)}\right)^{-1} \circ\left(T^{(k)}\right)^{-1}\right\}_{i=1}^{N} .
$$

The space $Q_{N}^{(k)}$ arises naturally if one considers shape optimization in Lagrange
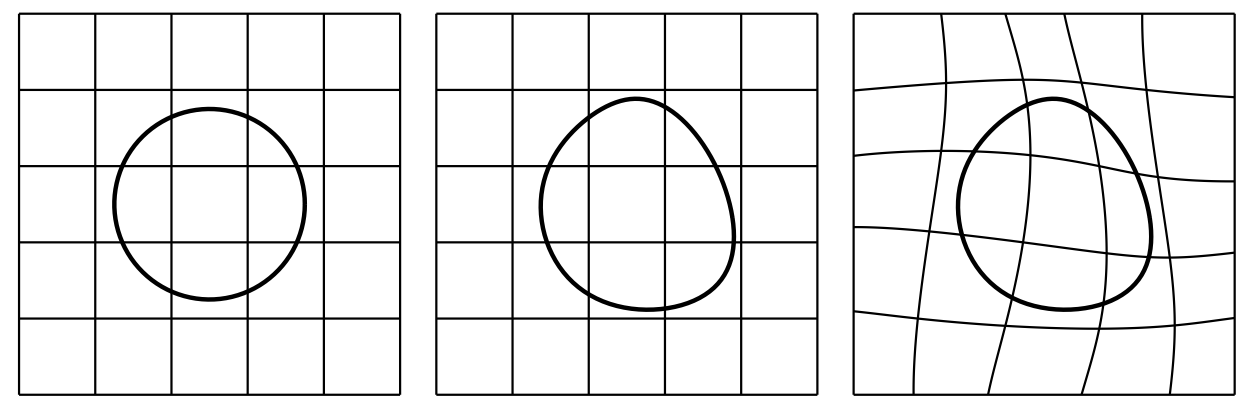

FIG. 1. Graphical example of a domain $\Omega^{0}$, a discretization $Q_{N}$, a perturbed domain $\Omega^{(1)}$, and the discretization $Q_{N}^{(1)}$. We assume that $Q_{N}$ is constructed on a grid. Left: initial configuration. Center: the domain is deformed according to a transformation $F^{(1)}$ whereas the basis functions $\mathbf{q}_{i}$ 's of $Q_{N}$ are constructed on the initial regular grid. Right: both the domain and the grid evolve according to $F^{(1)}$; the basis functions $\mathbf{q}_{i}^{(1)}$ 's of $Q_{N}^{(1)}$ are constructed on the curved grid via pullback.

coordinates [30], and satisfies $Q_{N}^{(k)} \subset W^{1, \infty}\left(\mathbb{R}^{d}, \mathbb{R}^{d}\right)$. Compared to $Q_{N}$, this new space $Q_{N}^{(k)}$ has a great computational advantage: the previously computed matrix $\mathbf{I}_{h}$ corresponds to the representation of the interpolation operators

$$
\mathcal{I}_{h}: Q_{N} \rightarrow\left(V_{h}\left(\Omega^{0}\right)\right)^{d}
$$


as well as to the representation of

$$
\mathcal{I}_{h}^{(k)}: Q_{N}^{(k)} \rightarrow\left(V_{h}\left(\Omega^{(k)}\right)\right)^{d}
$$

with respect to the basis $\left\{\mathbf{q}_{i}^{(k)}\right\}_{i=1}^{N}$ and $\left\{\mathbf{v}_{i}^{(k)}\right\}_{i=1}^{M}$. This implies that the vector $\mathbf{d} \tilde{\mathbf{J}}_{\mathbf{q}^{(k)}}^{(k)}$ (the right-hand side of (30)) can be assembled more easily and quickly than $\mathbf{d} \tilde{\mathbf{J}}_{\mathbf{q}}^{(k)}$. On top of that, (33) motivates the interpretation (30). Note that for given test cases, the asymptotic rate of convergence in this approximation can be explicitely computed.

Once the load vector for the update step has been assembled, we need to assemble the stiffness matrix. In principle, this should be done as well with respect to the new discretization (31). Alternatively, one can redefine the inner product of $\mathcal{X}$ so that

$$
\left(\mathbf{q}_{i}, \mathbf{q}_{j}\right)_{\mathcal{X}}=\left(\mathbf{q}_{i}^{(k)}, \mathbf{q}_{j}^{(k)}\right)_{\mathcal{X}^{(k)}} \text { for all } i, j=1, \ldots, N,
$$

and the stiffness matrix can be computed once and for all in the initialization step.

Finally, one computes the vector $\mathbf{d t}^{(k)}$, which contains the coefficients of the series expansion of $d T^{(k)}$ with respect to the basis of (31). Computing $\mathbf{I}_{h} \mathbf{d t}^{(k)}$ returns the coefficients of the interpolant of $d T^{(k)}$ in $\left(V_{h}\left(\Omega^{(k)}\right)\right)^{d}$. Due to the isoparametric nature of its basis $\left\{\mathbf{v}_{i}^{(k)}\right\}_{i=1}^{M}$, these coefficients equal those resulting from interpolating $d T^{(k)} \circ F^{(k)}$ onto $\left(V\left(\Omega^{0}\right)\right)^{d}$. This explains why (28) is the algebraic counterpart of (26).

Step 4-5. The simplest way to compute $s$ is by line search. In this case, we have to evaluate $J$ on $T^{(k)}+s d T^{(k)}$ for various $s$. In light of (28), this means computing the state variable $u$ using isoparametric finite elements whose coefficients are

$$
\mathbf{f}_{s}^{(k+1)}=\mathbf{f}^{(k)}+s \mathbf{I}_{h} \mathbf{d} \mathbf{t}^{(k)},
$$

and choosing $s$ such that the value of $J$ is minimal. Of course, one has to enforce admissibility of $T^{(k)}+s d T^{(k)}$. By Theorem 3.2, it is sufficient to verify that the minimum of

$$
\operatorname{det}\left(\mathbf{D}\left(F^{(k)}+s \mathcal{I}_{h}\left(d T^{(k)}\right)\right)\right) \approx \operatorname{det}\left(\mathbf{D}\left(T^{(k)}+s d T^{(k)}\right)\right)
$$

remains positive. However, note that small values of (34) may negatively affect the ellipticity constant of the BVP (2b), which in turn negatively affects the constant of finite element error estimates. Finally, one rescales $d T^{(k)}:=s d T^{(k)}$, set $T^{(k+1)}=$ $\left(\mathcal{I}+\mathrm{d} T^{(k)}\right) \circ\left(T^{(k)}\right)$, and goes back to step 2 .

7. Numerical experiments. We split our numerical investigations in two parts. In the first one, we consider a PDE-constrained shape optimization problem that admits stable minimizers. We use this test case to investigate the approximation properties of the algorithm presented in section 6 for different discretizations of control and state variables. In the second part, we test our approach on more challenging shape optimization problems for which analytical solutions are unavailable. The numerical results are obtained with a code based on the finite element library Firedrake $[4,5,7,8,14,28,36,41]$.

7.1. Bernoulli free-boundary problem. We consider the Dirichlet BVP

$$
-\Delta u=0 \text { in } \Omega, \quad u=u_{\text {in }} \quad \text { on } \partial \Omega^{\text {in }}, \quad u=u_{\text {out }} \text { on } \partial \Omega^{\text {out }},
$$

stated on the domain $\Omega \subset \mathbb{R}^{2}$ depicted in Figure 2. The goal is to find the shape of $\partial \Omega^{\text {in }}$ so that the Neumann trace $\frac{\partial u}{\partial \mathbf{n}}$ is equal to a prescribed function $g$ on $\partial \Omega^{\text {in }}$. For 


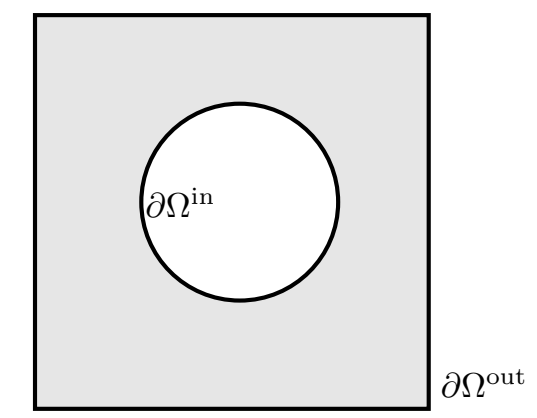

FIG. 2. Computational domain for the Dirichlet BVP (35). The external boundary $\partial \Omega^{\text {out }}$ is a square centered at the origin with a corner in $(1,1)$. The internal boundary $\partial \Omega^{\text {in }}$ is optimized to achieve $\left.\frac{\partial u}{\partial \mathbf{n}}\right|_{\partial \Omega^{\text {in }}}=g$.

the sake of simplicity, we assume that the Dirichlet data $u_{\text {in }}$ and the Neumann data $g$ are constants, and that only $\partial \Omega^{\text {in }}$ is "free" to move.

This Bernoulli free boundary problem can be reformulated as the following shape optimization problem [17]

$$
\inf _{\Omega \in \mathcal{U}_{\text {ad }}} \mathcal{J}(\Omega, u)=\int_{\Omega} \nabla u \cdot \nabla u+g^{2} \mathrm{~d} \boldsymbol{x}, \quad \text { subject to }\left\{\begin{array}{rlrl}
-\Delta u & =0 & & \text { in } \Omega, \\
u & =u_{\text {in }} & & \text { on } \partial \Omega^{\text {in }}, \\
u & =u_{\text {out }} & & \text { on } \partial \Omega^{\text {out }},
\end{array},\right.
$$

whose Fréchet derivative reads [30]

$d J(T ; \mathcal{T})=\int_{T\left(\Omega_{0}\right)} \operatorname{div} \mathcal{T}\left(\nabla u_{T\left(\Omega_{0}\right)} \cdot \nabla u_{T\left(\Omega_{0}\right)}+g^{2}\right)-\nabla u_{T\left(\Omega_{0}\right)} \cdot\left(\mathbf{D} \mathcal{T}+\mathbf{D} \mathcal{T}^{\top}\right) \nabla u_{T\left(\Omega_{0}\right)} \mathrm{d} \boldsymbol{x}$.

In [17], the authors have studied this shape optimization problem (36) in detail and, performing shape analysis in polar coordinates, have shown that the shape Hessian is both continuous and coercive (when restricted to normal perturbations) in the $H^{1 / 2}(\partial \Omega)$-norm. For this reason, minimizers of (36) are stable.

To construct a test case for our numerical simulations, we set the optimal shape of $\partial \Omega^{\text {in }}$ to be a circle centered at the origin with radius 0.4 . For such a choice of $\partial \Omega^{\text {in }}$, the function (expressed in polar coordinates)

$$
u(r, \varphi)=\ln (0.4)-\log (r)
$$

satisfies the Dirichlet BVP (35) with $u_{\text {in }}=0$ and $u_{\text {out }}=u$. The Neumann data on the interior boundary is $g=2.5$. The value $J_{\min }$ of the misfit functional in the optimal shape is approximatively 28.306941614057237 . This value has been computed with quadratic isoparametric finite elements on a sequence of nested meshes; the relative error between the value of the misfit functional computed on the last and on the second last mesh is approximately $6 \cdot 10^{-11}$.

As initial guess, we set $\partial \Omega_{0}^{\text {in }}$ to be a circle of radius 0.5 centered at $(0.04,0.05)$. Note that we have repeated these numerical experiments with other 3 choices for the initial guess $\partial \Omega_{0}^{\text {in }}$ and have obtained similar results. These alternative initial guesses are: a circle of radius 0.47 centered at $(0.07,0.03)$, a circle of radius 0.55 centered at $(-0.1,0)$, and a circle of radius 0.5367 centered at $(-0.137,0.03)$.

To discretize geometric transformations, we consider linear/quadratic/cubic tensorized Schoenberg B-splines constructed on regular grids [46]. These grids are refined 
uniformly (with widths ranging from $1.8 \times 2^{-1}$ to $1.8 \times 2^{-6}$ ) and are contained in a square (the hold-all domain $D$ ) that is centered at the origin and has a corner at $(0.95,0.95)$, so that $\partial \Omega^{\text {out }}$ is not modified in the optimization process. Finite element approximations of $u_{T\left(\Omega_{0}\right)}$ are computed with linear/quadratic isoparametric finite elements on a sequence of 5 triangular meshes generated using uniform refinement in Gmsh [23]. Note that finer meshes are adjusted to fit curved boundaries.

The optimization is carried out by repeating the following simple procedure for a fixed number of iterations: at every iteration, we compute a $H_{0}^{1}(D)$-descent direction $d T$ by solving (18) and choose the optimization step $s \in\{0,0.1, \ldots, 1\}$ that minimizes $J(T+s d T \circ T)$. Such a simple optimization strategy is sufficient for our numerical experiments, although we are aware that it is not efficient. The development of more efficient optimization strategies in the context of shape optimization is a current topic of research. In [45], the authors obtained promising results with a BFGStype algorithm based on a Steklov-Poincaré metric. We defer to future research the numerical comparison of optimization strategies for shape optimization.

In Figure 3, we plot two steps of this simple optimization strategy. Transformations are discretized with quadratic B-splines built on the fourth grid, whereas the state $u_{T\left(\Omega_{0}\right)}$ is approximated with linear finite elements on the second coarsest mesh. Qualitatively, we observe the expected behavior of a (truncated) linesearch. The predicted-descent line is given by $J(\mathcal{T})-d J(\mathcal{T}, s d T)$, with $s=0,0.1,0.2,0.3$, and is tangential to $J(T+s d T \circ T)$ at $s=0$. This shows empirically that formula (37) is correct.

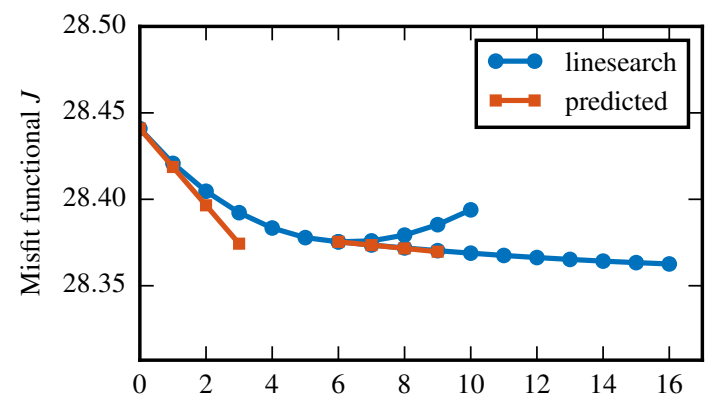

FIG. 3. Evolution of $J$ on two optimization steps. The second linesearch starts at the minimum of the first one. The predicted descent is computed evaluating $d J$ on the (selected) descent direction.

Next, we investigate to which accuracy we can solve shape optimization problems. In particular, we study the impact of the discretization (polynomial-)degree and (refinement-)level used for the control and the state. This is done systematically by keeping certain discretization parameters fixed and varying the remaining ones. To simplify the exposition, we associate the term grid to the discretization of the control (that is, of geometric transformations), whereas the term mesh refers to the discretization of the state).

First, we fix the control discretization to quadratic B-splines built on the finest grid. For each finite element mesh (previously generated with Gmsh), we perform 101 steps of our simple optimization strategy employing linear FE approximations of the state $u_{T\left(\Omega_{0}\right)}$. Although not displayed, the sequence of shapes always converges qualitatively to the optimum. For a quantitative comparison, we store the minimum 
of $J$ for every linesearch and plot the absolute error with respect to $J_{\min }$ in Figure 4 (we plot a convergence history for each mesh). Henceforth, we use the notation Jerr to refer to this absolute error. We observe that the convergence history lines
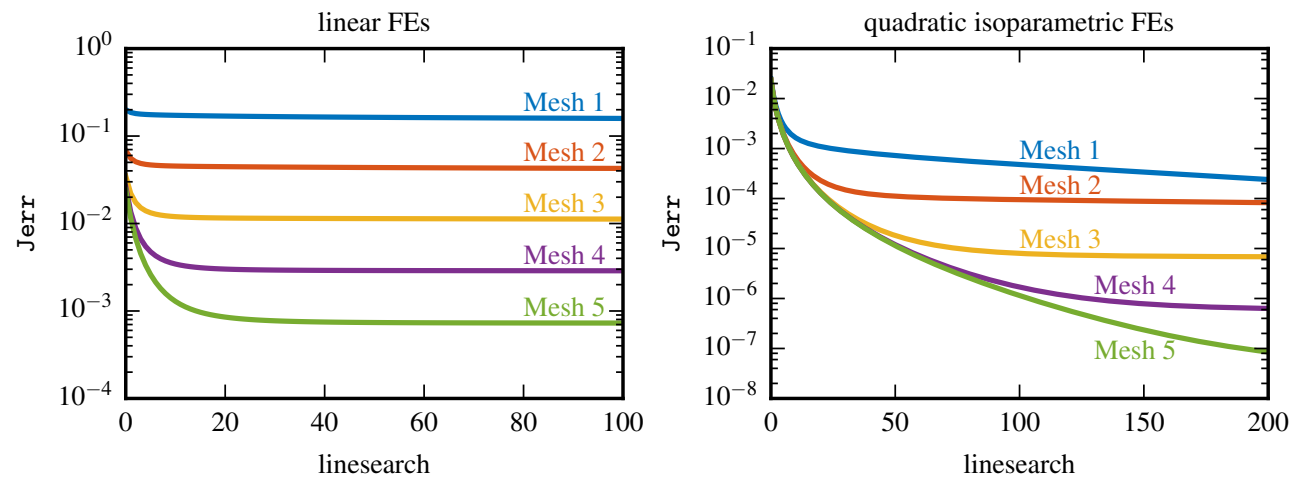

FIG. 4. Evolution of Jerr when the state variable is discretized with linear (left) and quadratic (right) FEs on a different meshes constructed via uniform refinement. Convergence lines saturate at different levels, which decay algebraically with respect to the mesh width.

saturate at different levels. In particular, the saturation level decays algebraically with respect to the mesh width. To further investigate the impact of $\mathrm{FE}$ approximations on shape optimization, we repeat this experiment with quadratic isoparamentric FEs. Again, we observe algebraic convergence with respect to the mesh width, but at higher convergence rate (note the difference in the y-axis scale). In order to reach the saturation level on finer meshes, more optimization steps have to be carried out. This issue has been observed in [30] as well, and is probably due to the simplicity of the optimization strategy. Before proceeding further, let us point out that the saturation level worsens if quadratic isoparametric FEs are replaced by quadratic affine FEs; see Figure 5.

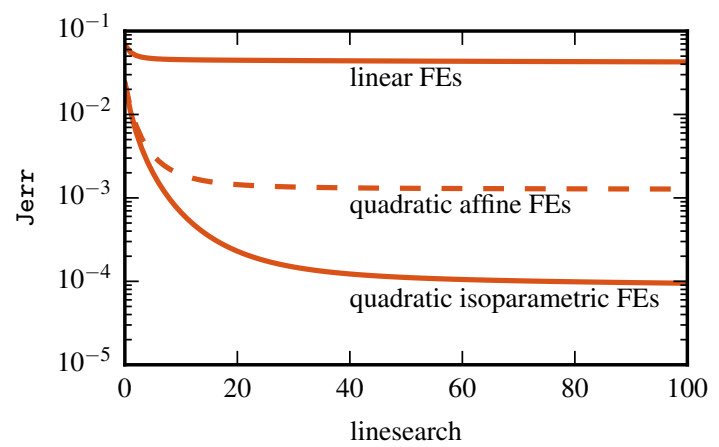

FIG. 5. Evolution of Jerr when the state variable is discretized with linear, quadratic affine, and quadratic isoparametric FEs on the second mesh. Quadratic affine FEs perform worse than their isoparametric counterpart.

In the previous experiments, we kept the discretization of transformations fixed. Now, we test different discretizations. In Figure 6, we consider two different discretization degrees of the control variable (linear/cubic B-splines). For each of these 
discretization degrees, we consider 6 grids and 5 meshes. The state is approximated once with linear and once with quadratic isoparametric FEs. For each combination, we plot Jerr after 200 iterations. We observe that both the discretization of the control and of the state have an impact on Jerr (the algebraic decay with respect to the FE mesh width is conspicuous).
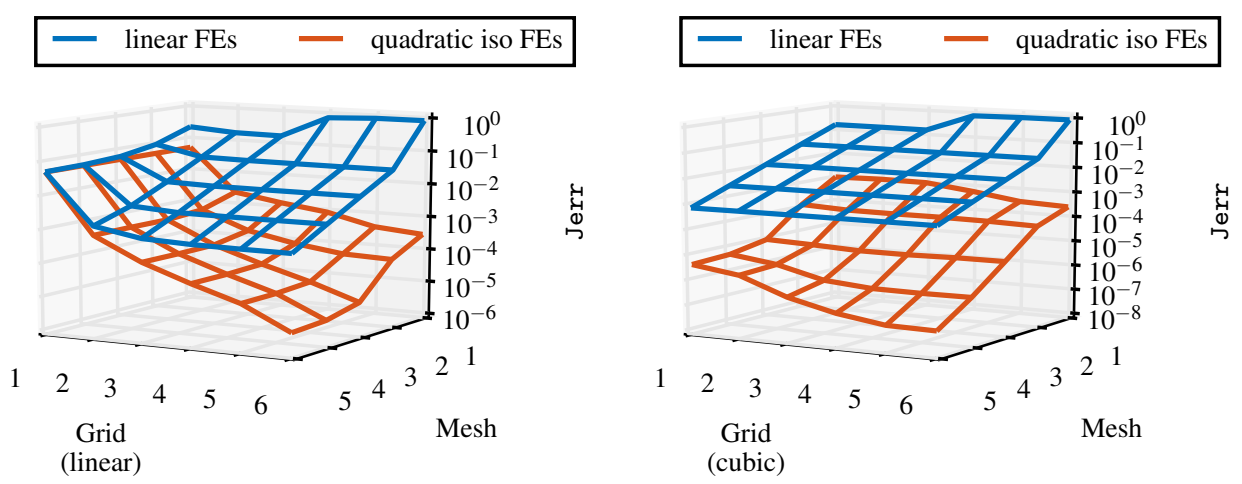

FIG. 6. Value of Jerr after 201 optimization steps for different combination of control and state discretization. The algebraic rate of convergence with respect to mesh refinement and the benefits of higher-degree discretizations of transformations are clearly visible.

In Figure 7 (left), we consider the finest level and highest degree of the control discretization. We plot Jerr (after 200 iterations) versus the FE mesh index and consider both linear and quadratic isoparametric FE approximations of the state. The algebraic rates of convergence for linear and quadratic FEs read 1.97 and 3.24, respectively. This rates are in line with our expectations because duality techniques can be employed to prove superconvergence in the FE approximation of the quadratic functional $J$ [31]. Figure 6 shows also that Jerr is almost entirely dominated by the
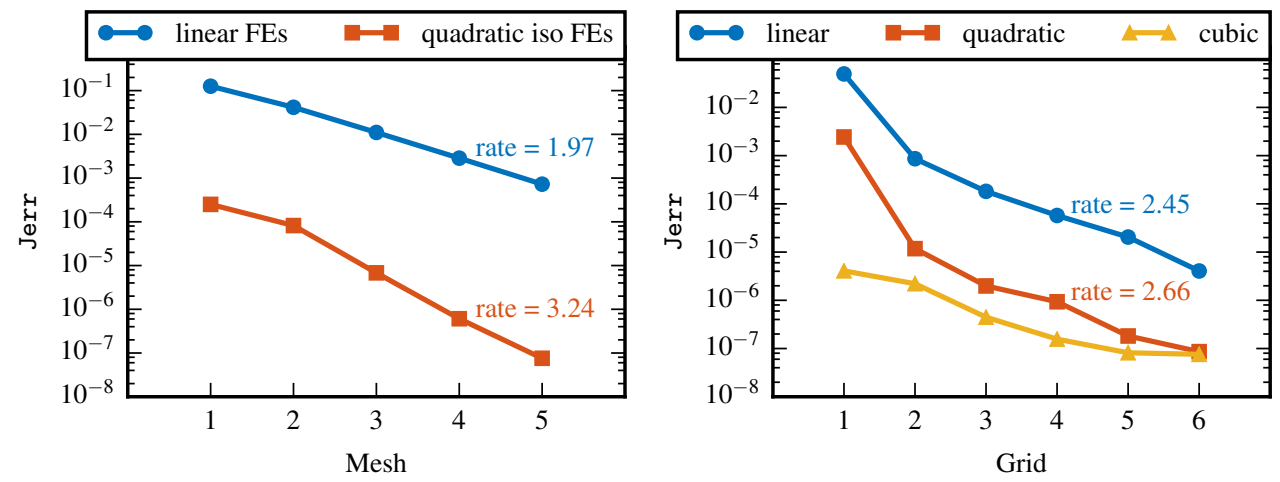

FIG. 7. Details from Figure 6: Left: convergence with respect to FE discretization of the state. Right: convergence with respect to B-spline discretization of the control.

error in the state when transformations are discretized with cubic B-splines. This is better highlighted in Figure 7 (right). There, we fix the state discretization to quadratic isoparametric FEs on the finest mesh and plot Jerr versus the grid index 
for linear, quadratic, and cubic B-splines. For instance, grid refinement for cubic B-splines has a very mild impact on Jerr (due to the FE approximation error of the state). We observe that the algebraic convergence rate for grid refinement of linear B-splines is approximately 2.45. This rate is higher than our expectations (a perturbation analysis via the Strang lemma would give rise to only linear convergence).

Finally, we consider the highest discretization level of the control and investigate whether its discretization degree has an impact on the rate of convergence of Jerr with respect to the $\mathrm{FE}$ discretization of the state. Let us recall that the regularity of the state on a perturbed domain depends, in principle, on the regularity of the domain. When perturbed with less smooth transformations, the resulting domain may not guarantee that the regularity of the state is preserved. This may have a negative impact on the FE approximation. In Figure 8, we plot Jerr versus FE mesh refinement (both for linear and quadratic FEs) when transformations are discretized with linear B-splines. When the state is approximated with quadratic isoparametric FEs, the control discretization error is negligible only on much finer grids. However, it seems that the $\mathrm{FE}$ convergence rate is not affected by the the discretization degree of the control (in Figure 7, left, the control is discretized with cubic B-splines, and a similar convergence rate is observed).

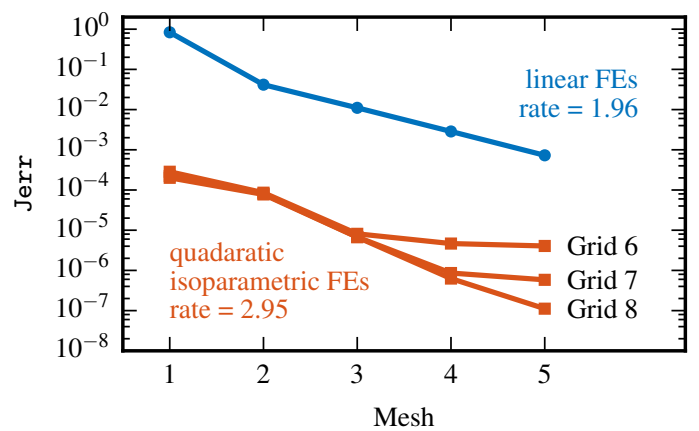

FIG. 8. Surprisingly, discretizing transformations with linear B-splines instead of cubic does not affect the rate of convergence of Jerr with respect to FE discretization of the state (compare with Figure 7, left).

7.2. Energy minimization in Stokes flow. Next, we consider the shape optimization of a $2 \mathrm{D}$ obstacle $\Omega_{o}$ embedded in a viscous fluid $\Omega_{c}$ (see Figure 9). This problem has been thoroughly investigated in [44]. The state variables are the velocity $\mathbf{u}$ and the pressure $p$ of the fluid. These are governed by Stokes' equations, whose weak formulation reads: find $\mathbf{u} \in H^{1}\left(\Omega_{c} ; \mathbb{R}^{2}\right)$ and $p \in L_{0}^{2}\left(\Omega_{c}\right)=\left\{p \in L^{2}\left(\Omega_{c}\right): \int_{\Omega_{c}} p \mathrm{~d} \boldsymbol{x}=0\right\}$ such that $\left.\mathbf{u}\right|_{\Gamma_{\text {in }}}=\mathbf{g},\left.u\right|_{\Gamma_{\text {wall }}}=0$ and

$$
\int_{\Omega_{c}} \sum_{i=1}^{2} \nabla u_{i} \cdot \nabla v_{i}-p \operatorname{div} \mathbf{v}+q \operatorname{div} \mathbf{u} \mathrm{d} \boldsymbol{x}=0
$$

for all $\mathbf{v} \in H^{1}\left(\Omega_{c} ; \mathbb{R}^{2}\right)$ and $q \in L_{0}^{2}\left(\Omega_{c}\right)$ such that $\left.\mathbf{v}\right|_{\Gamma_{\text {in }}}=0$ and $\left.\mathbf{v}\right|_{\Gamma_{\text {wall }}}=0$. It is known that (38) admits a unique solution if the computational domain $\Omega_{c}$ is Lipschitz $[26,49]$.

The energy dissipated in the fluid due to shear forces is given by

$$
\mathcal{J}\left(\Omega_{c}, \mathbf{u}, p\right)=\int_{\Omega_{c}} \sum_{i=1}^{2} \nabla u_{i}^{\top} \nabla u_{i} \mathrm{~d} \boldsymbol{x} \equiv \int_{\Omega_{c}}\|\mathbf{D u}\|_{F}^{2} \mathrm{~d} \boldsymbol{x},
$$




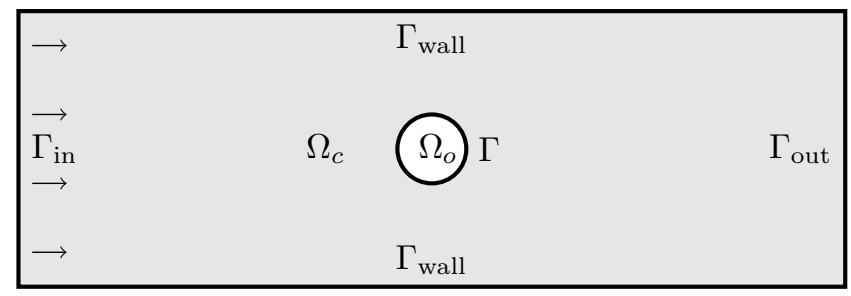

FIG. 9. Computational domain for the Stokes BVP (38). The external boundary comprises: the inflow boundary $\Gamma_{\mathrm{in}}$, the outflow boundary $\Gamma_{\mathrm{out}}$, the obstacle boundary $\Gamma$, and the wall boundary $\Gamma_{\text {wall }}$. The obstacle boundary is a circle centered at the origin with radius 0.5 , whereas the remaining boundary is a rectangle with width 12 and height 5.

and its shape derivative is given $b^{3}$

$$
\begin{aligned}
d \mathcal{J}(T, \mathcal{T})=\int_{T\left(\Omega_{c}\right)} \sum_{i=1}^{2}\left(\nabla u_{i}^{\top} \nabla u_{i} \operatorname{div} \mathcal{T}\right. & \left.-\nabla u_{i}^{\top}\left(\mathbf{D} \mathcal{T}^{\top}+\mathbf{D} \mathcal{T}\right) \nabla u_{i}\right) \\
& +2 \operatorname{tr}(\mathbf{D u D} \mathcal{T}) p-2 p \operatorname{div} \mathbf{u} \operatorname{div} \mathcal{T} \mathrm{d} \boldsymbol{x}
\end{aligned}
$$

Minimizing (39) subject to (38) alone is problematic because energy dissipation can be reduced by shrinking or removing the obstacle. Therefore, we introduce two additional constraints: we require the area and the barycentre of the obstacle to remain constant. Similarly to [44], we define the functionals

$$
\begin{aligned}
\mathcal{A}(T) & :=\int_{T\left(\Omega_{c}\right)} 1 \mathrm{~d} \boldsymbol{x}-\int_{\Omega_{c}} 1 \mathrm{~d} \boldsymbol{x}, \\
\mathcal{B}_{i}(T) & :=\int_{T\left(\Omega_{c}\right)} x_{i} \mathrm{~d} \boldsymbol{x}-\int_{\Omega_{c}} x_{i} \mathrm{~d} \boldsymbol{x}, \text { for } i=1,2 .
\end{aligned}
$$

For the sake of simplicity, we enforce the constraints $\mathcal{A}(T)=0$ and $\mathcal{B}_{i}(T)=0$, $i=1,2$ onto the shape optimization problem in the form of penalty functions, that is, we replace the functional (39) with

$$
\mathcal{J}_{p}(T)=\mathcal{J}(T)+\frac{\mu_{0}}{2} \mathcal{A}^{2}(T)+\sum_{i=1}^{2} \frac{\mu_{i}}{2} \mathcal{B}_{i}^{2}(T),
$$

where $\mathcal{A}^{2}(T):=(\mathcal{A}(T))^{2}, \mathcal{B}_{i}^{2}(T):=\left(\mathcal{B}_{i}(T)\right)^{2}$, and $0 \leq \mu_{i} \in \mathbb{R}, \mathrm{i}=0,1,2$. The shape derivatives of the squared constraints are given by

$$
\begin{aligned}
& d A^{2}(T, \mathcal{T})=2 \mathcal{A}(T) \int_{T\left(\Omega_{c}\right)} \operatorname{div} \mathcal{T} \mathrm{d} \boldsymbol{x}, \\
& d B_{i}^{2}(T, \mathcal{T})=2 \mathcal{B}_{i}(T) \int_{T\left(\Omega_{c}\right)} \operatorname{div}\left(x_{i} \mathcal{T}\right) \mathrm{d} \boldsymbol{x}, \text { for } i=1,2 .
\end{aligned}
$$

The state variables are discretized with Taylor-Hood P2-P1 finite elements on a triangular mesh. This discretization is stable [16]. The resulting linear system can then be solved using GMRES and a block-diagonal preconditioner based on the stiffness matrix and on the mass matrix for the velocity- and the pressure-block, respectively [16]. The control is discretized with cubic B-splines on a rectangular grid

\footnotetext{
${ }^{3}$ We believe that this volume based formula (which can be computed with [42]) is already known to the shape optimization community, although we did not manage to find it explicitly in available publications.
} 

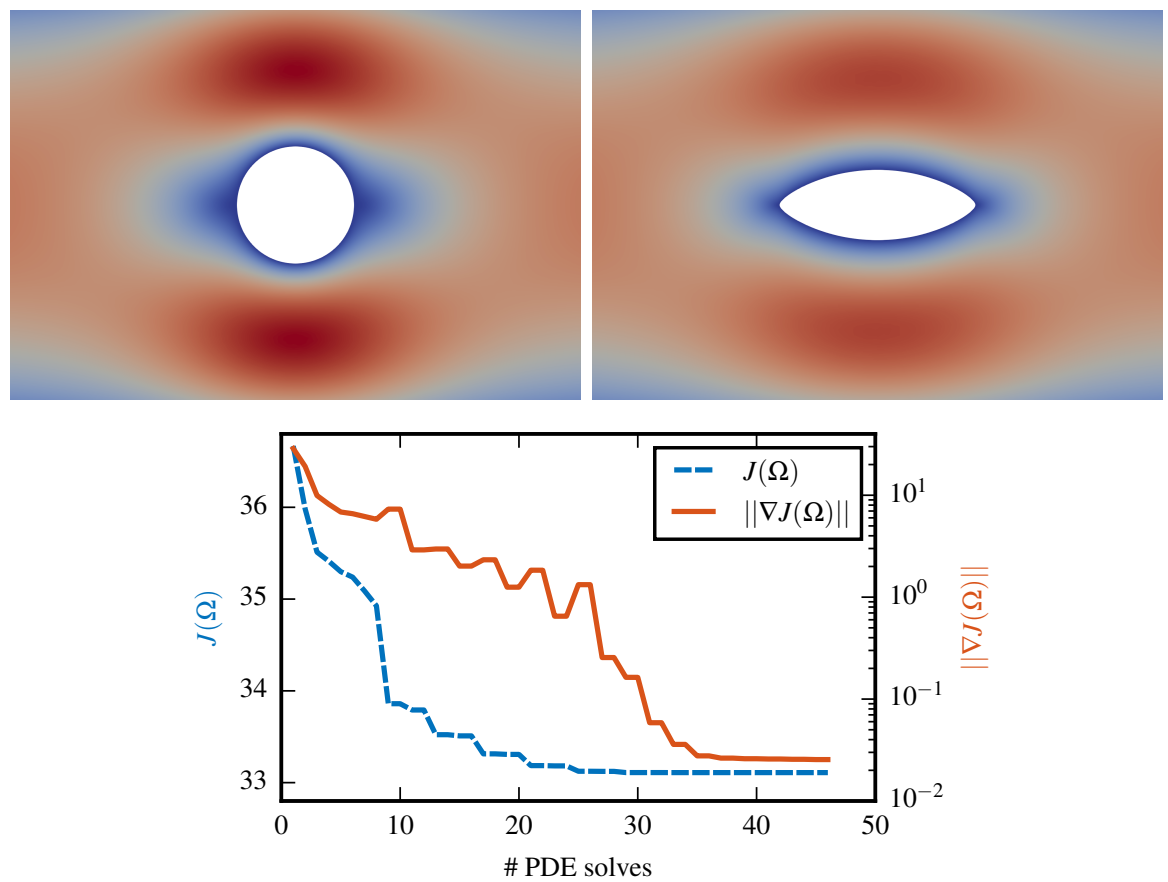

FIG. 10. Left: initial shape. Right: optimized shape using quadratic B-splines. The colour indicates the magnitude of the velocity field. Bottom: convergence history; the gradient is measured using the $H^{1}(D)$-norm.

that covers the entire channel. We can see that the optimized shape in Figure 10 is qualitatively similar to the one obtained by [44], although the shape optimization algorithm used in [44] relies on the boundary based formulation of (40). For this example we have used a simple steepest descent with line search; the number of optimization steps can be drastically reduced employing more sophisticated optimization algorithms [44].

7.3. Compliance minimization under linear elasticity. We conclude this section on numerical experiments with another classical example from shape optimization: compliance minimization of a cantilever subject to a given load (see Figure 11). The structural behaviour of the cantilever is modelled by linear elasticity. In particular, we consider the following variational problem: find $\mathbf{u} \in H^{1}\left(\Omega ; \mathbb{R}^{2}\right)$ such that $\left.\mathbf{u}\right|_{\Gamma_{1}}=0$ and

$$
\int_{\Omega}(A e(\mathbf{u})): \nabla \mathbf{v} \mathrm{d} \boldsymbol{x}-\int_{\Gamma_{2}} \mathbf{g} \cdot \mathbf{v} \mathrm{d} S=0
$$

for all $\mathbf{v} \in H^{1}\left(\Omega ; \mathbb{R}^{2}\right)$ with $\left.\mathbf{v}\right|_{\Gamma_{1}}=0$. In (44), the symbol : denotes the Frobenius inner product of matrices, $e(\mathbf{u})=\operatorname{sym}(\nabla \mathbf{u})=\frac{1}{2}\left(\nabla \mathbf{u}+\nabla \mathbf{u}^{\top}\right)$ denotes the strain tensor, and $A$ encodes the Hookes' law of the material. It is well known that (44) admits a unique stable solution for compatible data $\mathbf{g}$ [13, Page 22]. In this numerical experiment, we minimize the compliance

$$
\mathcal{J}(\Omega, \mathbf{u})=\int_{\Omega}(A e(\mathbf{u})): e(\mathbf{u}) \mathrm{d} \boldsymbol{x},
$$




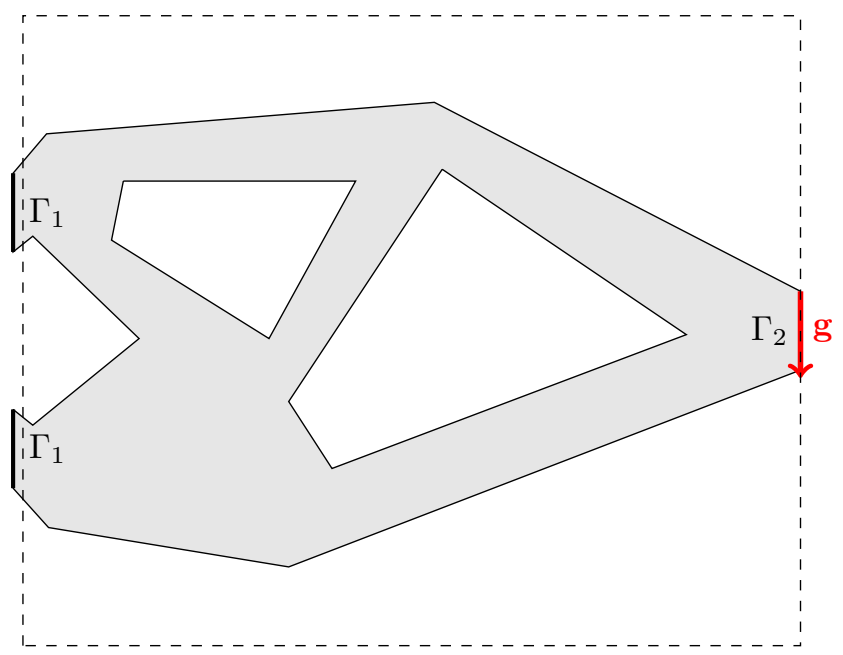

FIG. 11. Computational domain for the elasticity $B V P$ (44). On the two segments $\Gamma_{1}$ we impose homogeneous Dirichlet boundary conditions. On $\Gamma_{2}$ we impose $\mathbf{g}=(0,-1)^{\top}$ as Neumann boundary condition. The dashed rectangle indicated the hold-all domain $D$. We exclude the part close to the left boundary, as singularities occur in the solution to the PDE close to the corners.

whose shape derivative reads (the formula can be derived following closely [47, Thm 7.7])

$$
\begin{aligned}
d \mathcal{J}(T, \mathcal{T})= & \int_{T(\Omega)} A \operatorname{sym}(\mathbf{D u D} \mathcal{T}): \operatorname{sym}(\mathbf{D u}) \mathrm{d} \boldsymbol{x} \\
& +\int_{T(\Omega)} A \operatorname{sym}(\mathbf{D u}): \operatorname{sym}(\mathbf{D u D} \mathcal{T}) \mathrm{d} \boldsymbol{x} \\
& +\int_{T(\Omega)} A \operatorname{sym}(\mathbf{D u}): \operatorname{sym}(\mathbf{D u}) \operatorname{div} \mathcal{T} \mathrm{d} \boldsymbol{x} .
\end{aligned}
$$

Similarly to (42), we enforce a volume constraint by adding a penalty function to (45). We discretize the state variables with piecewise linear Lagrangian finite elements on a triangular mesh; the control is discretized with cubic B-splines on a rectangular grid covering the cantilever. We use the same Lamé parameters as in [3] $(E=15$, $\nu=0.35)$ and obtain a qualitatively similar shape, which is shown in Figure 12.

8. Conclusion. We have formulated shape optimization problems in terms of deformation diffeomorphisms. This perspective simplifies the treatment of PDEconstrained shape optimization problems because it couples naturally with isoparametric finite element discretization of the PDE-constraint. In particular, it retains the asymptotic behavior of higher-order FE discretization, and it allows the solution of PDE-constrained shape optimization problems to high accuracy, as confirmed by detailed numerical experiments. This shape optimization method can be implemented in standard finite element software and used to tackle challenging shape optimization problems that stem from industrial applications. The approach advocated is modular and can be combined with more advanced optimization algorithms, such as that of Schulz et al. [45]; research in this vein will form the basis of future work. 

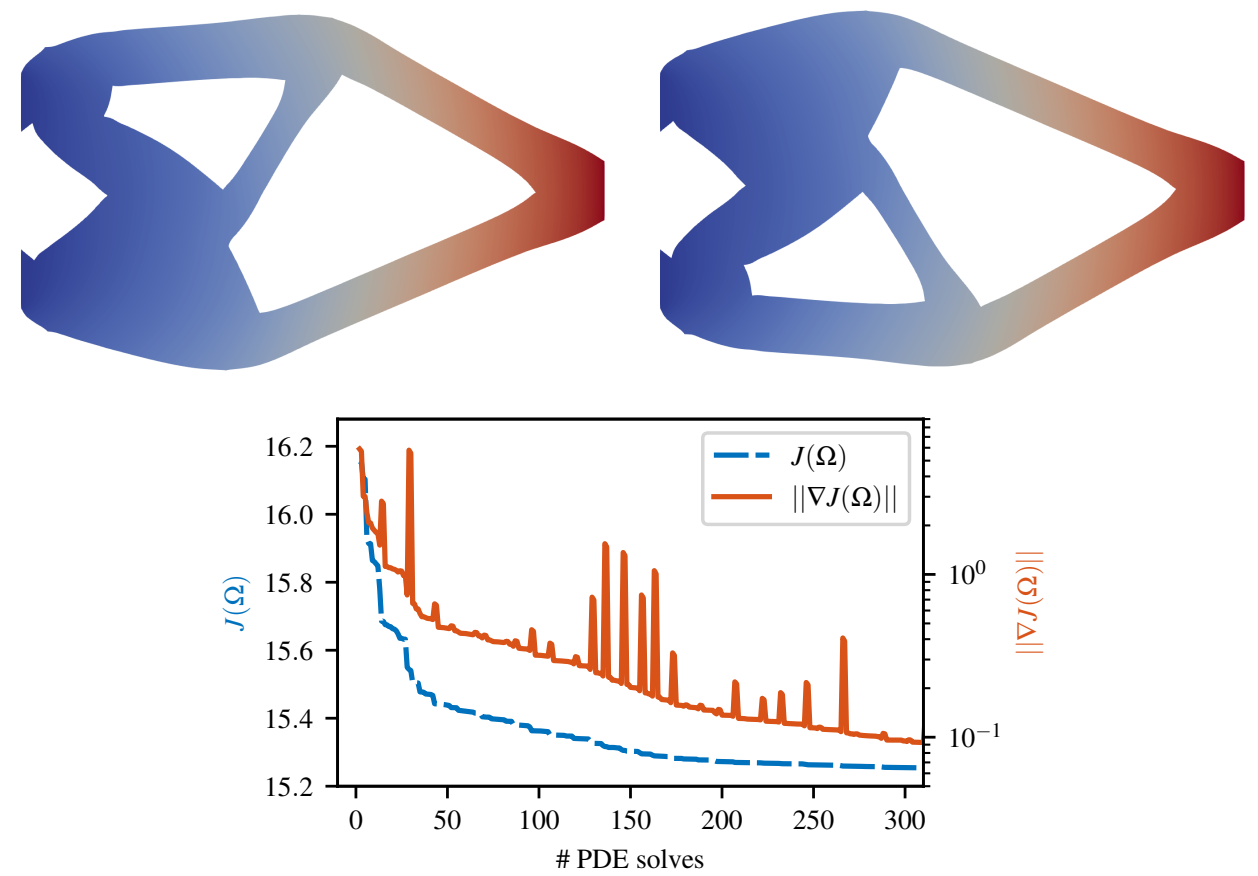

FIG. 12. Top left: Optimal shape using cubic B-splines. Top right: Optimal shape using as initial guess the domain obtained by flipping Figure 11 with respect to the horizontal axis. Bottom: Convergence history for the left shape; the gradient is measured using the $H^{1}(D)$-norm.

[1] G. Allaire, Conception optimale de structures, Springer-Verlag, Berlin, 2007.

[2] G. Allaire, F. Jouve, And A.-M. Toader, A level-set method for shape optimization, C. R. Math. Acad. Sci. Paris, 334 (2002), pp. 1125-1130.

[3] G. Allaire and O. Pantz, Structural optimization with FreeFem++, Struct. Multidiscip. Optim., 32 (2006), pp. 173-181.

[4] P. R. Amestoy, I. S. Duff, J.-Y. L'Excellent, And J. Koster, A fully asynchronous multifrontal solver using distributed dynamic scheduling, SIAM Journal on Matrix Analysis and Applications, 23 (2001), pp. 15-41.

[5] P. R. Amestoy, A. Guermouche, J.-Y. L'Excellent, and S. Pralet, Hybrid scheduling for the parallel solution of linear systems, Parallel Computing, 32 (2006), pp. 136-156.

[6] P. F. Antonietti, N. Bigoni, and M. Verani, Mimetic finite difference method for shape optimization problems, Springer International Publishing, Cham, 2015, pp. 125-132.

[7] S. Balay, S. Abhyankar, M. F. Adams, J. Brown, P. Brune, K. Buschelman, L. Dalcin, V. Eijkhout, W. D. Gropp, D. Kaushik, M. G. Knepley, L. C. McInnes, K. Rupp, B. F. Smith, S. Zampini, H. Zhang, and H. Zhang, PETSc users manual, Tech. Report ANL-95/11 - Revision 3.7, Argonne National Laboratory, 2016.

[8] S. Balay, W. D. Gropp, L. C. McInnes, and B. F. Smith, Efficient management of parallelism in object oriented numerical software libraries, in Modern Software Tools in Scientific Computing, E. Arge, A. M. Bruaset, and H. P. Langtangen, eds., Birkhäuser Press, 1997, pp. 163-202.

[9] K. Bandara, F. Cirak, G. Of, O. Steinbach, and J. Zapletal, Boundary element based multiresolution shape optimisation in electrostatics, J. Comput. Phys., 297 (2015), pp. 584598.

[10] A. Barton and S. Mayboroda, Boundary-value problems for higher-order elliptic equations in non-smooth domains, in Concrete Operators, Spectral Theory, Operators in Harmonic Analysis and Approximation, Springer, 2014, pp. 53-93.

[11] M. BergGren, A unified discrete-continuous sensitivity analysis method for shape optimization, in Applied and numerical partial differential equations, vol. 15 of Comput. Methods Appl. Sci., Springer, New York, 2010, pp. 25-39. 
[12] D. Braess, Finite elements, Cambridge University Press, Cambridge, third ed., 2007.

[13] P. G. Ciarlet, The finite element method for elliptic problems, Society for Industrial and Applied Mathematics (SIAM), Philadelphia, PA, 2002.

[14] L. D. Dalcin, R. R. Paz, P. A. Kler, And A. Cosimo, Parallel distributed computing using Python, Advances in Water Resources, 34 (2011), pp. 1124-1139.

[15] M. C. Delfour And J.-P. ZolÉsio, Shapes and geometries. Metrics, analysis, differential calculus, and optimization, Society for Industrial and Applied Mathematics (SIAM), Philadelphia, PA, second ed., 2011.

[16] H. C. Elman, D. J. Silvester, and A. J. Wathen, Finite elements and fast iterative solvers: with applications in incompressible fluid dynamics, Oxford University Press (UK), 2014.

[17] K. Eppler and H. Harbrecht, Efficient treatment of stationary free boundary problems, Appl. Numer. Math., 56 (2006), pp. 1326-1339.

[18] K. Eppler AND H. HARBrecht, Shape optimization for free boundary problems-analysis and numerics, in Constrained optimization and optimal control for partial differential equations, vol. 160 of Internat. Ser. Numer. Math., Birkhäuser/Springer Basel AG, Basel, 2012, pp. $277-288$.

[19] L. C. Evans, Partial differential equations, American Mathematical Society, Providence, RI, second ed., 2010.

[20] P. E. Farrell, D. A. Ham, S. W. Funke, and M. E. Rognes, Automated derivation of the adjoint of high-level transient finite element programs, SIAM J. Sci. Comput., 35 (2013), pp. C369-C393.

[21] M. Galewski and M. Radulescu, A note on a global invertibility of locally Lipschitz functions on $R^{n}$, ArXiv e-prints, (2015), https://arxiv.org/abs/1509.02965.

[22] H. Garcke, C. Hecht, M. Hinze, C. Kahle, and K. F. Lam, Shape optimization for surface functionals in Navier-Stokes flow using a phase field approach, Interfaces Free Bound., 18 (2016), pp. 219-261.

[23] C. Geuzaine and J.-F. Remacle, Gmsh: A 3-d finite element mesh generator with built-in preand post-processing facilities, International Journal for Numerical Methods in Engineering, 79 (2009), pp. 1309-1331.

[24] M. Giacomini, O. Pantz, and K. Trabelsi, Certified Descent Algorithm for shape optimization driven by fully-computable a posteriori error estimators, ArXiv e-prints, (2016), https://arxiv.org/abs/1604.04921.

[25] D. Gilbarg and N. S. Trudinger, Elliptic partial differential equations of second order, Classics in Mathematics, Springer-Verlag, Berlin, 2001.

[26] V. Girault and P.-A. Raviart, Finite element methods for Navier-Stokes equations: theory and algorithms, vol. 5, Springer Ser. Comput. Math, 1986.

[27] P. GRISvard, Elliptic problems in nonsmooth domains, vol. 69 of Classics in Applied Mathematics, Society for Industrial and Applied Mathematics (SIAM), Philadelphia, PA, 2011.

[28] B. HeNdRICKSON AND R. Leland, A multilevel algorithm for partitioning graphs, in Supercomputing '95: Proceedings of the 1995 ACM/IEEE Conference on Supercomputing, New York, 1995, ACM Press, p. 28.

[29] M. Hinze, R. Pinnau, M. Ulbrich, and S. Ulbrich, Optimization with PDE constraints, Springer, New York, 2009.

[30] R. Hiptmair and A. Paganini, Shape optimization by pursuing diffeomorphisms, Comput. Methods Appl. Math., 15 (2015), pp. 291-305.

[31] R. Hiptmair, A. Paganini, and S. Sargheini, Comparison of approximate shape gradients, BIT, 55 (2015), pp. 459-485.

[32] K. Höllig, Finite element methods with B-splines, Society for Industrial and Applied Mathematics (SIAM), Philadelphia, PA, 2003.

[33] R. C. James, Characterizations of reflexivity, Studia Math., 23 (1963/1964), pp. 205-216.

[34] G. Katriel, Mountain pass theorems and global homeomorphism theorems, Ann. Inst. H. Poincaré Anal. Non Linéaire, 11 (1994), pp. 189-209.

[35] A. Laurain and K. Sturm, Distributed shape derivative via averaged adjoint method and applications, ESAIM: M2AN, 50 (2016), pp. 1241-1267.

[36] F. Luporini, D. A. Ham, And P. H. J. Kelly, An algorithm for the optimization of finite element integration loops, Submitted to ACM TOMS, (2016), http://arxiv.org/abs/1604. 05872 .

[37] F. Murat and J. Simon, Etude de problemes d'optimal design, Springer Berlin Heidelberg, Berlin, Heidelberg, 1976, pp. 54-62.

[38] A. Paganini, Approximate shape gradients for interface problems, in New Trends in Shape Optimization, vol. 166 of Internat. Ser. Numer. Math., Springer International Publishing, 2015, pp. 217-227. 
[39] A. Paganini, Numerical shape optimization with finite elements, PhD thesis, ETH Zurich, 2016.

[40] A. Paganini and R. Hiptmair, Approximate Riesz representatives of shape gradients, in System Modeling and Optimization (CSMO 2015), 2017 (to appear).

[41] F. Rathgeber, D. A. Ham, L. Mitchell, M. Lange, F. Luporini, A. T. T. McRae, G.-T. Bercea, G. R. Markall, and P. H. J. Kelly, Firedrake: automating the finite element method by composing abstractions, ACM Trans. Math. Softw., 43 (2016), pp. 24:1-24:27.

[42] S. Schmidt, Automatic generation of Hadamard shape derivatives and approximate shape Hessians. submitted, 2016.

[43] S. Schmidt, C. Ilic, V. Schulz, and N. R. Gauger, Three-dimensional large-scale aerodynamic shape optimization based on shape calculus, AIAA journal, 51 (2013), pp. 2615-2627.

[44] V. Schulz And M. Siebenborn, Computational comparison of surface metrics for PDE constrained shape optimization, Computational Methods in Applied Mathematics, (2015).

[45] V. H. Schulz, M. Siebenborn, And K. Welker, Efficient PDE constrained shape optimization based on Steklov-Poincaré-type metrics, SIAM Journal on Optimization, 26 (2016), pp. $2800-2819$

[46] L. L. Schumaker, Spline functions, Society for Industrial and Applied Mathematics, Philadelphia, PA, 2015. Computational methods.

[47] K. Sturm, On shape optimization with non-linear partial differential equations, PhD thesis, Technische Universität Berlin, October 2014.

[48] K. Sturm, Convergence of Newton's method in shape optimisation via approximate normal functions, ArXiv e-prints, (2016), https://arxiv.org/abs/1608.02699.

[49] R. Temam, Navier-Stokes equations: theory and numerical analysis, vol. 343, American Mathematical Soc., 2001. 